\title{
Search for first-generation scalar and vector leptoquarks
}

V. M. Abazov, ${ }^{23}$ B. Abbott,${ }^{58}$ A. Abdesselam, ${ }^{11}$ M. Abolins, ${ }^{51}$ V. Abramov, ${ }^{26}$ B. S. Acharya, ${ }^{17}$ D. L. Adams, ${ }^{60}$ M. Adams, ${ }^{38}$ S. N. Ahmed, ${ }^{21}$ G. D. Alexeev, ${ }^{23}$ G. A. Alves ${ }^{2}$ N. Amos,${ }^{50}$ E. W. Anderson, ${ }^{43}$ Y. Arnoud, ${ }^{9}$ M. M. Baarmand, ${ }^{55}$ V. V. Babintsev, ${ }^{26}$ L. Babukhadia, ${ }^{55}$ T. C. Bacon, ${ }^{28}$ A. Baden, ${ }^{47}$ B. Baldin, ${ }^{37}$ P. W. Balm, ${ }^{20}$ S. Banerjee, ${ }^{17}$ E. Barberis, ${ }^{30}$ P. Baringer, ${ }^{44}$ J. Barreto, ${ }^{2}$ J. F. Bartlett, ${ }^{37}$ U. Bassler, ${ }^{12}$ D. Bauer, ${ }^{28}$ A. Bean, ${ }^{44}$ M. Begel, ${ }^{54}$ A. Belyaev, ${ }^{35}$ S. B. Beri, ${ }^{15}$ G. Bernardi, ${ }^{12}$ I. Bertram, ${ }^{27}$ A. Besson, ${ }^{9}$ R. Beuselinck, ${ }^{28}$ V. A. Bezzubov, ${ }^{26}$ P. C. Bhat ${ }^{37}$ V. Bhatnagar, ${ }^{11}$

M. Bhattacharjee, ${ }^{55}$ G. Blazey, ${ }^{39}$ S. Blessing, ${ }^{35}$ A. Boehnlein, ${ }^{37}$ N. I. Bojko, ${ }^{26}$ F. Borcherding, ${ }^{37}$ K. Bos, ${ }^{20}$ A. Brandt, ${ }^{60}$

R. Breedon, ${ }^{31}$ G. Briskin, ${ }^{59}$ R. Brock, ${ }^{51}$ G. Brooijmans, ${ }^{37}$ A. Bross, ${ }^{37}$ D. Buchholz, ${ }^{40}$ M. Buehler, ${ }^{38}$ V. Buescher, ${ }^{14}$ V. S. Burtovoi, ${ }^{26}$ J. M. Butler, ${ }^{48}$ F. Canelli, ${ }^{54}$ W. Carvalho, ${ }^{3}$ D. Casey, ${ }^{51}$ Z. Casilum, ${ }^{55}$ H. Castilla-Valdez, ${ }^{19}$

D. Chakraborty, ${ }^{39}$ K. M. Chan, ${ }^{54}$ S. V. Chekulaev, ${ }^{26}$ D. K. Cho, ${ }^{54}$ S. Choi, ${ }^{34}$ S. Chopra,${ }^{56}$ J. H. Christenson, ${ }^{37}$ M. Chung, ${ }^{38}$ D. Claes, ${ }^{52}$ A. R. Clark, ${ }^{30}$ J. Cochran, ${ }^{34}$ L. Coney, ${ }^{42}$ B. Connolly, ${ }^{35}$ W. E. Cooper, ${ }^{37}$ D. Coppage, ${ }^{44}$ S. Crépé-Renaudin, ${ }^{9}$ M. A. C. Cummings, ${ }^{39}$ D. Cutts, ${ }^{59}$ G. A. Davis, ${ }^{54}$ K. Davis ${ }^{29}$ K. De, ${ }^{60}$ S. J. de Jong, ${ }^{21}$ K. Del Signore, ${ }^{50}$ M. Demarteau, ${ }^{37}$ R. Demina, ${ }^{45}$ P. Demine, ${ }^{9}$ D. Denisov, ${ }^{37}$ S. P. Denisov, ${ }^{26}$ S. Desai, ${ }^{55}$ H. T. Diehl,${ }^{37}$ M. Diesburg, ${ }^{37}$ G. Di Loreto, ${ }^{51}$

S. Doulas, ${ }^{49}$ P. Draper, ${ }^{60}$ Y. Ducros, ${ }^{13}$ L. V. Dudko, ${ }^{25}$ S. Duensing, ${ }^{21}$ L. Duflot, ${ }^{11}$ S. R. Dugad, ${ }^{17}$ A. Duperrin, ${ }^{10}$ A. Dyshkant, ${ }^{39}$ D. Edmunds, ${ }^{51}$ J. Ellison, ${ }^{34}$ V. D. Elvira, ${ }^{37}$ R. Engelmann, ${ }^{55}$ S. Eno, ${ }^{47}$ G. Eppley, ${ }^{62}$ P. Ermolov, ${ }^{25}$ O. V. Eroshin, ${ }^{26}$

J. Estrada, ${ }^{54}$ H. Evans, ${ }^{53}$ V. N. Evdokimov, ${ }^{26}$ T. Fahland, ${ }^{33}$ S. Feher, ${ }^{37}$ D. Fein, ${ }^{29}$ T. Ferbel, ${ }^{54}$ F. Filthaut, ${ }^{21}$ H. E. Fisk, ${ }^{37}$ Y. Fisyak, ${ }^{56}$ E. Flattum, ${ }^{37}$ F. Fleuret, ${ }^{30}$ M. Fortner, ${ }^{39}$ H. Fox ${ }^{40}$ K. C. Frame, ${ }^{51}$ S. Fu, ${ }^{53}$ S. Fuess, ${ }^{37}$ E. Gallas,${ }^{37}$ A. N. Galyaev, ${ }^{26}$ M. Gao, ${ }^{53}$ V. Gavrilov, ${ }^{24}$ R. J. Genik II, ${ }^{27}$ K. Genser, ${ }^{37}$ C. E. Gerber, ${ }^{38}$ Y. Gershtein, ${ }^{59}$ R. Gilmartin, ${ }^{35}$ G. Ginther, ${ }^{54}$

B. Gómez, ${ }^{5}$ G. Gómez, ${ }^{47}$ P. I. Goncharov, ${ }^{26}$ J. L. González Solís, ${ }^{19}$ H. Gordon, ${ }^{56}$ L. T. Goss, ${ }^{61}$ K. Gounder, ${ }^{37}$ A. Goussiou, ${ }^{28}$ N. Graf, ${ }^{56}$ G. Graham, ${ }^{47}$ P. D. Grannis, ${ }^{55}$ J. A. Green, ${ }^{43}$ H. Greenlee, ${ }^{37}$ S. Grinstein, ${ }^{1}$ L. Groer, ${ }^{53}$ S. Grünendahl, ${ }^{37}$

A. Gupta, ${ }^{17}$ S. N. Gurzhiev, ${ }^{26}$ G. Gutierrez, ${ }^{37}$ P. Gutierrez, ${ }^{58}$ N. J. Hadley, ${ }^{47}$ H. Haggerty, ${ }^{37}$ S. Hagopian, ${ }^{35}$ V. Hagopian, ${ }^{35}$ R. E. Hall, ${ }^{32}$ P. Hanlet, ${ }^{49}$ S. Hansen, ${ }^{37}$ J. M. Hauptman, ${ }^{43}$ C. Hays, ${ }^{53}$ C. Hebert, ${ }^{44}$ D. Hedin, ${ }^{39}$ J. M. Heinmiller, ${ }^{38}$

A. P. Heinson, ${ }^{34}$ U. Heintz, ${ }^{48}$ T. Heuring, ${ }^{35}$ M. D. Hildreth, ${ }^{42}$ R. Hirosky, ${ }^{63}$ J. D. Hobbs,${ }^{55}$ B. Hoeneisen, ${ }^{8}$ Y. Huang, ${ }^{50}$

R. Illingworth, ${ }^{28}$ A. S. Ito, ${ }^{37}$ M. Jaffré, ${ }^{11}$ S. Jain, ${ }^{17}$ R. Jesik, ${ }^{28}$ K. Johns, ${ }^{29}$ M. Johnson, ${ }^{37}$ A. Jonckheere, ${ }^{37}$ M. Jones, ${ }^{36}$ H. Jöstlein, ${ }^{37}$ A. Juste, ${ }^{37}$ W. Kahl, ${ }^{45}$ S. Kahn, ${ }^{56}$ E. Kajfasz, ${ }^{10}$ A. M. Kalinin, ${ }^{23}$ D. Karmanov, ${ }^{25}$ D. Karmgard, ${ }^{42}$

Z. Ke, ${ }^{4}$ R. Kehoe, ${ }^{51}$ A. Khanov, ${ }^{45}$ A. Kharchilava, ${ }^{42}$ S. K. Kim, ${ }^{18}$ B. Klima, ${ }^{37}$ B. Knuteson, ${ }^{30}$ W. Ko, ${ }^{31}$ J. M. Kohli, ${ }^{15}$

A. V. Kostritskiy, ${ }^{26}$ J. Kotcher, ${ }^{56}$ B. Kothari, ${ }^{53}$ A. V. Kotwal, ${ }^{53}$ A. V. Kozelov, ${ }^{26}$ E. A. Kozlovsky, ${ }^{26}$ J. Krane, $^{43}$ M. R. Krishnaswamy, ${ }^{17}$ P. Krivkova, ${ }^{6}$ S. Krzywdzinski, ${ }^{37}$ M. Kubantsev, ${ }^{45}$ S. Kuleshov, ${ }^{24}$ Y. Kulik, ${ }^{55}$ S. Kunori, ${ }^{47}$

A. Kupco, ${ }^{7}$ V. E. Kuznetsov, ${ }^{34}$ G. Landsberg, ${ }^{59}$ W. M. Lee, ${ }^{35}$ A. Leflat, ${ }^{25}$ C. Leggett, ${ }^{30}$ F. Lehner, ${ }^{37, *}$ J. Li, ${ }^{60}$ Q. Z. Li ${ }^{37}$ X. Li, ${ }^{4}$ J. G. R. Lima, ${ }^{3}$ D. Lincoln, ${ }^{37}$ S. L. Linn, ${ }^{35}$ J. Linnemann, ${ }^{51}$ R. Lipton, ${ }^{37}$ A. Lucotte, ${ }^{9}$ L. Lueking, ${ }^{37}$

C. Lundstedt, ${ }^{52}$ C. Luo, ${ }^{41}$ A. K. A. Maciel,${ }^{39}$ R. J. Madaras, ${ }^{30}$ V. L. Malyshev, ${ }^{23}$ V. Manankov, ${ }^{25}$ H. S. Mao, ${ }^{4}$ T. Marshall, ${ }^{41}$ M. I. Martin, ${ }^{39}$ R. D. Martin, ${ }^{38}$ K. M. Mauritz, ${ }^{43}$ B. May, ${ }^{40}$ A. A. Mayorov, ${ }^{41}$ R. McCarthy, ${ }^{55}$ T. McMahon, ${ }^{57}$

H. L. Melanson, ${ }^{37}$ M. Merkin, ${ }^{25}$ K. W. Merritt, ${ }^{37}$ C. Miao, ${ }^{59}$ H. Miettinen, ${ }^{62}$ D. Mihalcea, ${ }^{39}$ C. S. Mishra, ${ }^{37}$ N. Mokhov, ${ }^{37}$

N. K. Mondal, ${ }^{17}$ H. E. Montgomery, ${ }^{37}$ R. W. Moore, ${ }^{51}$ M. Mostafa, ${ }^{1}$ H. da Motta, ${ }^{2}$ E. Nagy, ${ }^{10}$ F. Nang, ${ }^{29}$ M. Narain, ${ }^{48}$

V. S. Narasimham, ${ }^{17}$ H. A. Neal, ${ }^{50}$ J. P. Negret, ${ }^{5}$ S. Negroni, ${ }^{10}$ T. Nunnemann, ${ }^{37}$ D. O’ Neil, ${ }^{51}$ V. Oguri, ${ }^{3}$ B. Olivier, ${ }^{12}$ N. Oshima, ${ }^{37}$ P. Padley, ${ }^{62}$ L. J. Pan, ${ }^{40}$ K. Papageorgiou, ${ }^{38}$ A. Para, ${ }^{37}$ N. Parashar, ${ }^{49}$ R. Partridge, ${ }^{59}$ N. Parua, ${ }^{55}$

M. Paterno, ${ }^{54}$ A. Patwa, ${ }^{55}$ B. Pawlik, ${ }^{22}$ J. Perkins, ${ }^{60}$ M. Peters,${ }^{36}$ O. Peters, ${ }^{20}$ P. Pétroff,,${ }^{11}$ R. Piegaia, ${ }^{1}$ B. G. Pope, ${ }^{51}$

E. Popkov, ${ }^{48}$ H. B. Prosper, ${ }^{35}$ S. Protopopescu, ${ }^{56}$ J. Qian, ${ }^{50}$ R. Raja, ${ }^{37}$ S. Rajagopalan, ${ }^{56}$ E. Ramberg, ${ }^{37}$ P. A. Rapidis, ${ }^{37}$

N. W. Reay, ${ }^{45}$ S. Reucroft, ${ }^{49}$ M. Ridel, ${ }^{11}$ M. Rijssenbeek, ${ }^{55}$ F. Rizatdinova, ${ }^{45}$ T. Rockwell, ${ }^{51}$ M. Roco, ${ }^{37} \mathrm{P}$. Rubinov, ${ }^{37}$

R. Ruchti, ${ }^{42}$ J. Rutherfoord, ${ }^{29}$ B. M. Sabirov, ${ }^{23}$ G. Sajot, ${ }^{9}$ A. Santoro, ${ }^{2}$ L. Sawyer, ${ }^{46}$ R. D. Schamberger, ${ }^{55}$ H. Schellman, ${ }^{40}$

A. Schwartzman, ${ }^{1}$ N. Sen, ${ }^{62}$ E. Shabalina, ${ }^{38}$ R. K. Shivpuri, ${ }^{16}$ D. Shpakov, ${ }^{49}$ M. Shupe, ${ }^{29}$ R. A. Sidwell, ${ }^{45}$ V. Simak, ${ }^{7}$

H. Singh, ${ }^{34}$ J. B. Singh, ${ }^{15}$ V. Sirotenko, ${ }^{37}$ P. Slattery, ${ }^{54}$ E. Smith, ${ }^{58}$ R. P. Smith, ${ }^{37}$ R. Snihur, ${ }^{40}$ G. R. Snow, ${ }^{52}$ J. Snow, ${ }^{57}$ S. Snyder, ${ }^{56}$ J. Solomon, ${ }^{38}$ V. Sorín, ${ }^{1}$ M. Sosebee, ${ }^{60}$ N. Sotnikova, ${ }^{25}$ K. Soustruznik, ${ }^{6}$ M. Souza, ${ }^{2}$ N. R. Stanton, ${ }^{45}$

G. Steinbrück, ${ }^{53}$ R. W. Stephens, ${ }^{60}$ F. Stichelbaut,${ }^{56}$ D. Stoker ${ }^{33}$ V. Stolin, ${ }^{24}$ A. Stone, ${ }^{46}$ D. A. Stoyanova, ${ }^{26}$ M. Strauss, ${ }^{58}$

M. Strovink, ${ }^{30}$ L. Stutte, ${ }^{37}$ A. Sznajder, ${ }^{3}$ M. Talby, ${ }^{10}$ W. Taylor, ${ }^{55}$ S. Tentindo-Repond, ${ }^{35}$ S. M. Tripathi, ${ }^{31}$

T. G. Trippe, ${ }^{30}$ A. S. Turcot, ${ }^{56}$ P. M. Tuts, ${ }^{53}$ P. van Gemmeren, ${ }^{37}$ V. Vaniev, ${ }^{26}$ R. Van Kooten, ${ }^{41}$ N. Varelas, ${ }^{38}$

L. S. Vertogradov, ${ }^{23}$ F. Villeneuve-Seguier, ${ }^{10}$ A. A. Volkov, ${ }^{26}$ A. P. Vorobiev, ${ }^{26}$ H. D. Wahl, ${ }^{35}$ H. Wang, ${ }^{40}$ Z.-M. Wang, ${ }^{55}$

J. Warchol, ${ }^{42}$ G. Watts, ${ }^{64}$ M. Wayne, ${ }^{42}$ H. Weerts, ${ }^{51}$ A. White,${ }^{60}$ J. T. White, ${ }^{61}$ D. Whiteson, ${ }^{30}$ J. A. Wightman, ${ }^{43}$

D. A. Wijngaarden, ${ }^{21}$ S. Willis, ${ }^{39}$ S. J. Wimpenny, ${ }^{34}$ J. Womersley, ${ }^{37}$ D. R. Wood, ${ }^{49}$ R. Yamada, ${ }^{37}$ P. Yamin, ${ }^{56}$ T. Yasuda, ${ }^{37}$ Y. A. Yatsunenko, ${ }^{23}$ K. Yip, ${ }^{56}$ S. Youssef, ${ }^{35}$ J. Yu, ${ }^{37}$ Z. Yu, ${ }^{40}$ M. Zanabria, ${ }^{5}$ H. Zheng, ${ }^{42}$ Z. Zhou, ${ }^{43}$ M. Zielinski, ${ }^{54}$ D. Zieminska, ${ }^{41}$ A. Zieminski, ${ }^{41}$ V. Zutshi, ${ }^{56}$ E. G. Zverev, ${ }^{25}$ and A. Zylberstejn ${ }^{13}$

(DØ Collaboration)

${ }^{1}$ Universidad de Buenos Aires, Buenos Aires, Argentina

${ }^{2}$ LAFEX, Centro Brasileiro de Pesquisas Físicas, Rio de Janeiro, Brazil

${ }^{3}$ Universidade do Estado do Rio de Janeiro, Rio de Janeiro, Brazil

${ }^{4}$ Institute of High Energy Physics, Beijing, People's Republic of China

${ }^{5}$ Universidad de los Andes, Bogotá, Colombia

${ }^{6}$ Charles University, Center for Particle Physics, Prague, Czech Republic 
${ }^{7}$ Institute of Physics, Academy of Sciences, Center for Particle Physics, Prague, Czech Republic

${ }^{8}$ Universidad San Francisco de Quito, Quito, Ecuador

${ }^{9}$ Institut des Sciences Nucléaires, IN2P3-CNRS, Universite de Grenoble 1, Grenoble, France

${ }^{10} C P P M$, IN2P3-CNRS, Université de la Méditerranée, Marseille, France

${ }^{11}$ Laboratoire de l'Accélérateur Linéaire, IN2P3-CNRS, Orsay, France

${ }^{12}$ LPNHE, Universités Paris VI and VII, IN2P3-CNRS, Paris, France

${ }^{13}$ DAPNIA/Service de Physique des Particules, CEA, Saclay, France

${ }^{14}$ Universität Mainz, Institut für Physik, Mainz, Germany

${ }^{15}$ Panjab University, Chandigarh, India

${ }^{16}$ Delhi University, Delhi, India

${ }^{17}$ Tata Institute of Fundamental Research, Mumbai, India

${ }^{18}$ Seoul National University, Seoul, Korea

${ }^{19}$ CINVESTAV, Mexico City, Mexico

${ }^{20}$ FOM-Institute NIKHEF and University of Amsterdam/NIKHEF, Amsterdam, The Netherlands

${ }^{21}$ University of Nijmegen/NIKHEF, Nijmegen, The Netherlands

${ }^{22}$ Institute of Nuclear Physics, Kraków, Poland

${ }^{23}$ Joint Institute for Nuclear Research, Dubna, Russia

${ }^{24}$ Institute for Theoretical and Experimental Physics, Moscow, Russia

${ }^{25}$ Moscow State University, Moscow, Russia

${ }^{26}$ Institute for High Energy Physics, Protvino, Russia

${ }^{27}$ Lancaster University, Lancaster, United Kingdom

${ }^{28}$ Imperial College, London, United Kingdom

${ }^{29}$ University of Arizona, Tucson, Arizona 85721

${ }^{30}$ Lawrence Berkeley National Laboratory and University of California, Berkeley, California 94720

${ }^{31}$ University of California, Davis, California 95616

${ }^{32}$ California State University, Fresno, California 93740

${ }^{33}$ University of California, Irvine, California 92697

${ }^{34}$ University of California, Riverside, California 92521

${ }^{35}$ Florida State University, Tallahassee, Florida 32306

${ }^{36}$ University of Hawaii, Honolulu, Hawaii 96822

${ }^{37}$ Fermi National Accelerator Laboratory, Batavia, Illinois 60510

${ }^{38}$ University of Illinois at Chicago, Chicago, Illinois 60607

${ }^{39}$ Northern Illinois University, DeKalb, Illinois 60115

${ }^{40}$ Northwestern University, Evanston, Illinois 60208

${ }^{41}$ Indiana University, Bloomington, Indiana 47405

${ }^{42}$ University of Notre Dame, Notre Dame, Indiana 46556

${ }^{43}$ Iowa State University, Ames, Iowa 50011

${ }^{44}$ University of Kansas, Lawrence, Kansas 66045

${ }^{45}$ Kansas State University, Manhattan, Kansas 66506

${ }^{46}$ Louisiana Tech University, Ruston, Louisiana 71272

${ }^{47}$ University of Maryland, College Park, Maryland 20742

${ }^{48}$ Boston University, Boston, Massachusetts 02215

${ }^{49}$ Northeastern University, Boston, Massachusetts 02115

${ }^{50}$ University of Michigan, Ann Arbor, Michigan 48109

${ }^{51}$ Michigan State University, East Lansing, Michigan 48824

${ }^{52}$ University of Nebraska, Lincoln, Nebraska 68588

${ }^{53}$ Columbia University, New York, New York 10027

${ }^{54}$ University of Rochester, Rochester, New York 14627

${ }^{55}$ State University of New York, Stony Brook, New York 11794

${ }^{56}$ Brookhaven National Laboratory, Upton, New York 11973

${ }^{57}$ Langston University, Langston, Oklahoma 73050

${ }^{58}$ University of Oklahoma, Norman, Oklahoma 73019

${ }^{59}$ Brown University, Providence, Rhode Island 02912

${ }^{60}$ University of Texas, Arlington, Texas 76019

${ }^{61}$ Texas A\&M University, College Station, Texas 77843

${ }^{62}$ Rice University, Houston, Texas 77005

${ }^{63}$ University of Virginia, Charlottesville, Virginia 22901

${ }^{64}$ University of Washington, Seattle, Washington 98195

(Received 23 May 2001; published 9 October 2001) 
We describe a search for the pair production of first-generation scalar and vector leptoquarks in the $e e j j$ and $e \nu j j$ channels by the $\mathrm{D} \emptyset$ Collaboration. The data are from the $1992-1996 p \bar{p}$ run at $\sqrt{s}=1.8 \mathrm{TeV}$ at the Fermilab Tevatron collider. We find no evidence for leptoquark production; in addition, no kinematically interesting events are observed using relaxed selection criteria. The results from the $e e j j$ and $e \nu j j$ channels are combined with those from a previous $\mathrm{D} \emptyset$ analysis of the $\nu \nu j j$ channel to obtain $95 \%$ confidence level (C.L.) upper limits on the leptoquark pair-production cross section as a function of mass and of $\beta$, the branching fraction to a charged lepton. These limits are compared to next-to-leading-order theory to set $95 \%$ C.L. lower limits on the mass of a first-generation scalar leptoquark of 225,204 , and $79 \mathrm{GeV} / c^{2}$ for $\beta=1, \frac{1}{2}$, and 0, respectively. For vector leptoquarks with gauge (Yang-Mills) couplings, 95\% C.L. lower limits of 345, 337 , and $206 \mathrm{GeV} / c^{2}$ are set on the mass for $\beta=1, \frac{1}{2}$, and 0 , respectively. Mass limits for vector leptoquarks are also set for anomalous vector couplings.

DOI: 10.1103/PhysRevD.64.092004

PACS number(s): 14.80.-j, 13.85.Rm

\section{INTRODUCTION}

\section{A. Leptoquarks}

Leptoquarks (LQ's) are exotic particles that couple to both leptons and quarks and carry color, fractional electric charge, and both lepton and baryon numbers [1]. Although the pattern of three generations of doublets of quarks and leptons suggests leptoquarks as a possible reason for an underlying unity, they are not required in the standard model. Leptoquarks, however, do appear in composite models, technicolor theories, grand unified theories, and superstringinspired $\mathrm{E}_{6}$ models. They are not part of the minimal supersymmetric (SUSY) standard model, but can be accommodated in certain extended SUSY models. Leptoquarks can be scalar (spin 0) or vector (spin 1) particles. In many models, both baryon and lepton numbers are conserved, allowing low-mass leptoquarks to exist without mediating proton decay.

Leptoquarks with universal couplings to all flavors would give rise to flavor-changing neutral currents and are severely constrained by low-energy experiments. We therefore assume in our analysis that there is no intergenerational mixing and that, e.g., first-generation leptoquarks couple only to $e$ or $\nu_{e}$ and to $u$ or $d$ quarks. In most models containing leptoquarks, each leptoquark species has a fixed branching fraction to $l^{ \pm} q: \beta=1, \frac{1}{2}$ or 0 . Models with intergenerational mixing or extra fermions can have any value of $\beta$ between 0 and 1.

The H1 and ZEUS experiments at the $e^{ \pm} p$ collider HERA at DESY published lower limits on the mass of a firstgeneration leptoquark that depend on the unknown leptoquark-lepton-quark coupling, $\lambda$ [2-10]. Pair production of leptoquarks, nearly independent of the value of $\lambda$, could occur in $e^{+} e^{-}$collisions via a virtual $\gamma$ or $Z$ in the $s$-channel and in $p \bar{p}$ collisions via an intermediary gluon. Experiments at the CERN $e^{+} e^{-}$LEP collider [11-14] and at the Fermilab Tevatron [15-17] searched for leptoquark pair production and set lower limits on the masses of leptoquarks.

In February 1997, the $\mathrm{H} 1$ and ZEUS experiments reported an excess of events at high $Q^{2}[18,19]$. A possible interpretation of these events is the resonant production of firstgeneration leptoquarks at a mass $\left(M_{\mathrm{LQ}}\right)$ near $200 \mathrm{GeV} / c^{2}$

\footnotetext{
*Also at University of Zurich, Zurich, Switzerland.
}

[20]. Additional data collected in 1997 did not confirm this excess $[6,21]$. (For a recent review of leptoquark phenomenology and the status of leptoquark searches at HERA and the Tevatron, see Ref. [1].)

\section{B. Leptoquark production at the Tevatron}

At the Tevatron, pair production of leptoquarks can proceed through quark-antiquark annihilation (dominant for $M_{\mathrm{LQ}}>100 \mathrm{GeV} / c^{2}$ ) and through gluon fusion, and is therefore independent of the LQ- $e-q$ Yukawa coupling $\lambda$. Pair production of first-generation leptoquarks can result in three final states: two electrons and two jets $(e e j j)$; one electron, a neutrino, and two jets $(e \nu j j)$; or two neutrinos and two jets $(\nu \nu j j)$. The decay branching fractions in the eejj, evjj, and $\nu \nu j j$ channels are $\beta^{2}, 2 \beta(1-\beta)$, and $(1-\beta)^{2}$, respectively. The cross section for $p \bar{p} \rightarrow \mathrm{LQ} \overline{\mathrm{LQ}} \rightarrow e e j j$ is therefore proportional to $\beta^{2}$. We use the next-to-leading-order (NLO) calculation of the pair-production cross section of scalar leptoquarks [22] to compare our experimental results with theory. This calculation has a theoretical uncertainty of about $15 \%$ which corresponds to the variation of the renormalization scale $\mu$ used in the calculations between $\mu=2 M_{\mathrm{LQ}}$ and $\mu$ $=\frac{1}{2} M_{\mathrm{LQ}}$. For vector leptoquarks, NLO calculations are not yet available, and we therefore use the leading-order (LO) pair-production cross section [23]. We consider three gluon couplings: Yang-Mills gauge couplings $\left(\kappa_{G}=\lambda_{G}=0\right)$, minimal vector anomalous couplings $\left(\kappa_{G}=1\right.$ and $\left.\lambda_{G}=0\right)$, and the anomalous couplings that yield the minimum cross section for $150 \mathrm{GeV} / c^{2}$ leptoquarks at $\sqrt{s}=1.8 \mathrm{TeV}\left(\kappa_{G}=1.3\right.$ and $\left.\lambda_{G}=-0.21\right)[23]$.

\section{DØ DETECTOR AND TRIGGERING}

The $\mathrm{D} \emptyset$ detector is a general-purpose detector consisting of three major systems: a central tracking system, a uranium/ liquid-argon calorimeter, and a muon spectrometer. These are described in Ref. [24]. The features most relevant to this analysis are summarized below.

The central tracking system has a cylindrical vertex drift chamber, a transition-radiation detector, a cylindrical central drift chamber, and drift chambers in the forward regions. The tracking system is used to determine the longitudinal $(z)$ position of the $p \bar{p}$ interaction and to find tracks associated with electrons and muons. Information from the transitionradiation detector helps separate electrons from charged pions. The calorimeter consists of a central calorimeter (CC) 
that covers the detector pseudorapidity [25] region $\left|\eta_{\text {det }}\right|$ $<1.2$ and two end calorimeters (EC) that cover $1.5<\left|\eta_{\text {det }}\right|$ $<4$.2. Scintillation counters located in the intercryostat region provide information about jets for $1.2<\left|\eta_{\text {det }}\right|<1.5$. The electromagnetic (EM) and hadronic calorimeters are segmented into cells in pseudorapidity and azimuthal angle $(\phi)$ of size $\Delta \eta_{\text {det }} \times \Delta \phi=0.1 \times 0.1 \quad(0.05 \times 0.05$ at $\mathrm{EM}$ shower maximum).

The Main Ring synchrotron lies above the Tevatron beam line and passes through the outer section of the central calorimeter. Protons used for antiproton production pass through the Main Ring while the Tevatron is operating. Interactions in the Main Ring can cause spurious energy deposits in the calorimeter leading to false missing transverse energy $\left(\mathbb{E}_{T}\right)$ in collected events. Certain triggers are rejected when the protons are being injected into the Main Ring, every time the Main Ring beam passes through the detector, and during the subsequent "calorimeter recovery" period; other triggers are rejected during injection and when the proton bunch is present, but accepted during calorimeter recovery periods (called a "minimal" Main Ring veto). Since all events are tagged with the state of the Main Ring at the time of collection, this rejection can be performed offline for triggers relying on less restrictive Main Ring requirements.

$\mathrm{D} \emptyset$ employs a three-level trigger system. Level 0 uses scintillation counters near the beam pipe to detect an inelastic collision. Level 1 sums the EM energy in calorimeter towers of size $\Delta \eta_{\text {det }} \times \Delta \phi=0.2 \times 0.2$. Level 2 is a software trigger that forms clusters of calorimeter cells and applies preliminary requirements on the shower shape. Certain triggers also require energy clusters to be isolated.

\section{EVENT RECONSTRUCTION AND PARTICLE IDENTIFICATION}

The DØ reconstruction program, DØRECO, processes the triggered data into events with kinematic quantities and particle identification. This includes finding interaction vertices, tracks, and jets, and identifying electrons and muons, each with loose quality criteria to reject poorly-measured objects. Additional requirements are then applied for each analysis.

\section{A. Electron identification}

Electron identification for the $e e j j$ and $e \nu j j$ analyses is very similar. Electron candidates are first identified by finding isolated clusters of energy in the EM calorimeter. These EM clusters are required to be in the fiducial volume of the detector, i.e., $\left|\eta_{\text {det }}\right|<1.1$ (CC) or $1.5<\left|\eta_{\text {det }}\right|<2.5$ (EC). EM clusters with a matching track from the primary vertex are called electrons; those without a matching track are called trackless electrons. A track and an EM cluster in the CC match if the distance between the track and the EM cluster centroid is small,

$$
\sigma_{\mathrm{trk}}=\sqrt{\left(\frac{\Delta \phi}{\delta_{\Delta \phi}}\right)^{2}+\left(\frac{\Delta z}{\delta_{\Delta z}}\right)^{2}}<10,
$$

where $\Delta \phi$ is the azimuthal mismatch, $\Delta z$ is the mismatch along the beam direction, and $\delta_{x}$ is the resolution for the observable $x$. In the EC, $\Delta z$ is replaced by $\Delta r$, the mismatch transverse to the beam.

For the $e e j j$ analysis, at least one of the two electrons in an event is required to have a matching track. An electron track can be improperly reconstructed due to inefficiencies in the central tracking chambers or because of poor matching between the track and EM cluster caused by incorrect vertex information. Using trackless electrons restores some of this lost efficiency, but at the expense of increased background. They are not used in the $e \nu j j$ analysis.

For electron candidates with a matching track, we apply a likelihood test based on the following five variables:

(1) Agreement between the observed shower shape and that expected for an electromagnetic shower. This is computed using a 41-variable covariance matrix for energy deposition in the cells of the electromagnetic calorimeter $\left(H\right.$-matrix $\chi^{2}$ [26]).

(2) The ratio of the shower energy found in the EM calorimeter to the total shower energy, the electromagnetic energy fraction (EMF), is required to be that expected for an EM shower.

(3) A small track match significance, $\sigma_{\text {trk }}$, is required.

(4) The ionization $d E / d x$ along the track is required to be that for a single minimum-ionizing particle.

(5) A variable characterizing the energy deposited in the transition-radiation detector is required to be consistent with the expectation for an electron.

To a good approximation, these five quantities are independent of each other for electron showers. For EM objects without a matching track, an $H$-matrix $\chi^{2}<100$ is required.

All EM objects are required to have deposited most of their energy in the EM calorimeter $(E M F>0.9)$. We also require EM objects to be isolated, using the variable

$$
\mathcal{I} \equiv \frac{E_{\text {tot }}(\mathcal{R}=0.4)-E_{\mathrm{EM}}(\mathcal{R}=0.2)}{E_{\mathrm{EM}}(\mathcal{R}=0.2)},
$$

where $E_{\text {tot }}(\mathcal{R}=0.4)$ and $E_{\mathrm{EM}}(\mathcal{R}=0.2)$ are the total and $\mathrm{EM}$ energies in a cone of radius $\mathcal{R} \equiv \sqrt{(\Delta \eta)^{2}+(\Delta \phi)^{2}}=0.4$ or 0.2 centered on the EM cluster, where the pseudorapidity is measured with respect to the interaction vertex [25]. For electrons with matching tracks, we require $\mathcal{I}<0.15$. To reduce the multijet background by about $50 \%$ in dielectron data in which one electron does not have a matching track, we require that electron to have $\mathcal{I}<0.10$. The electron identification criteria are summarized in Table I.

The electron $E_{T}$ resolution is $\sigma\left(E_{T}\right) / E_{T}=0.0157$ $\oplus\left(0.072 \mathrm{GeV}^{1 / 2} / \sqrt{E_{T}}\right) \oplus 0.66 \mathrm{GeV} / E_{T}$, where $\oplus$ denotes a sum in quadrature. The resolution in $\eta$ and $\phi$ for an electron is excellent, less than $10^{-2}$ [27].

\section{B. Jet reconstruction}

Jet reconstruction [28] is based on energy deposition in calorimeter towers (the calorimeter cells within $\Delta \eta \times \Delta \phi$ $=0.1 \times 0.1)$ with $E_{T}>1 \mathrm{GeV}$. Starting with the highest- $E_{T}$ tower, the energy deposited in a cone of radius $\mathcal{R}=0.7$ around the center of the tower is summed and a new energy- 
TABLE I. Electron identification requirements.

\begin{tabular}{lcc}
\hline \hline Requirement & Electrons with tracks & Electrons without tracks \\
\hline Fiducial volume & $\left|\eta_{\text {det }}\right|<1.1$ or $1.5<\left|\eta_{\text {det }}\right|<2.5$ & $\left|\eta_{\text {det }}\right|<1.1$ or $1.5<\left|\eta_{\text {det }}\right|<2.5$ \\
Track match significance & $\sigma_{\text {trk }}<10$ & \\
Electromagnetic fraction & EMF $>0.9$ & EMF $>0.9$ \\
EM cluster isolation & $\mathcal{I}<0.15$ & $\mathcal{I}<0.10$ \\
EM cluster shape $H$-matrix & & $\chi^{2}<100$ \\
Five-variable likelihood & $<1.0$ & \\
\hline \hline
\end{tabular}

weighted center is determined. This procedure is repeated, using the new center, until the jet's direction is stable. Only jets with $E_{T}>8 \mathrm{GeV}$ are retained. The final direction of a jet is given by

$$
\begin{aligned}
& \theta_{\text {jet }}=\tan ^{-1}\left[\frac{\sqrt{\left(\sum_{i} E_{x}^{i}\right)^{2}+\left(\sum_{i} E_{y}^{i}\right)^{2}}}{\sum_{i} E_{z}^{i}}\right], \\
& \phi_{\text {jet }}=\tan ^{-1}\left(\frac{\sum_{i} E_{y}^{i}}{\sum_{i} E_{x}^{i}}\right), \\
& \eta_{\text {jet }}=-\ln \left(\tan \frac{\theta_{\text {jet }}}{2}\right)
\end{aligned}
$$

where the polar angle $\theta$ is measured relative to the interaction vertex, $E_{x}=E_{i} \sin \left(\theta_{i}\right) \cos \left(\phi_{i}\right), E_{y}=E_{i} \sin \left(\theta_{i}\right) \sin \left(\phi_{i}\right), E_{z}$ $=E_{i} \cos \left(\theta_{i}\right)$, and $i$ corresponds to all cells that are within $\mathcal{R}$ $=0.7$. Jets are required to have $\left|\eta_{\text {det }}\right|<2.5$ and EMF $<0.95$.

The measured jet energy is corrected for effects due to the underlying event and out-of-cone showering in the calorimeter. The transverse energy resolution for central jets $\left(\left|\eta_{\text {det }}\right|\right.$ $<0.5)$ varies from $\sigma\left(E_{T}\right) / E_{T}=0.154$ for $E_{T} \approx 36 \mathrm{GeV}$ to $\sigma\left(E_{T}\right) / E_{T}=0.050$ for $E_{T} \approx 300 \mathrm{GeV}$ [28]. The resolution in both $\eta$ and $\phi$ for $50 \mathrm{GeV}$ jets varies from approximately 0.02 for $\left|\eta_{\text {det }}\right|<0.5$ to approximately 0.06 for $2.0<\left|\eta_{\text {det }}\right|$ $<2.5$ and improves as the jet energy increases.

We use jets reconstructed with the large $\mathcal{R}=0.7$ cone size to decrease the number of final-state-radiation jets that are reconstructed separately from the parent jet and to improve the jet-energy and mass resolutions. Jets are ordered in descending value of $E_{T}$, with $j_{1}$, the leading jet, having the highest $E_{T}$.

\section{Missing transverse energy}

The missing transverse energy is calculated as

$$
\boldsymbol{E}_{T}=\sqrt{\mathbb{E}_{T_{x}}^{2}+\boldsymbol{E}_{T_{y}}^{2}}
$$

where

$$
\begin{aligned}
& \boldsymbol{E}_{T_{x}}=-\sum_{i} E_{i} \sin \left(\theta_{i}\right) \cos \left(\phi_{i}\right)-\sum_{j} \Delta E_{x}^{j}, \\
& \boldsymbol{E}_{T_{y}}=-\sum_{i} E_{i} \sin \left(\theta_{i}\right) \sin \left(\phi_{i}\right)-\sum_{j} \Delta E_{y}^{j} .
\end{aligned}
$$

The first sum is over all cells in the calorimeter and intercryostat detector above the noise threshold, and the second is over the corrections in $E_{T}$ applied to all electrons and jets in the event. The $\boldsymbol{E}_{T}$ resolution is approximately $4 \mathrm{GeV}$ per transverse component [29] and grows as the amount of calorimeter activity increases.

\section{Vertex finding}

The standard D $\emptyset$ vertex-finding algorithm uses tracks found in the central tracking system to locate the intersection of groups of tracks along the beam line. The group with the largest number of tracks is chosen as the primary vertex. However, since there is an average of 1.5 interactions per beam crossing, the hard-scattering vertex is not always chosen correctly by this algorithm. Using the electron to verify or recalculate the vertex significantly improves this efficiency [30]. The electron revertexing algorithm uses the track that best matches an EM calorimeter cluster and then recalculates the position of the vertex based on this track. The $z$ position of the vertex is calculated by fitting a straight line through the centroids of the EM cluster and the matching track. We require every event to contain at least one EM object with a matching track usable for revertexing. If both EM clusters have a matching track, the primary vertex is calculated based on information from both of them. The kinematic properties of the objects (electrons, jets, $\mathbb{E}_{T}$ ) in the event, such as transverse energy and pseudorapidity, are then recalculated based on the new vertex. All further analysis is done using the recalculated quantities.

Figure 1 illustrates the improvement in the resolution of the Z-boson mass, as well as the reduction in background due to vertex misidentification for $Z(\rightarrow e e)+2 j$ events, after the revertexing. Events in this plot are allowed to have one EM cluster without an associated track.

\section{SEARCH STRATEGIES AND OPTIMIZATION}

The choice of variables, and the selection of their optimal values, for improving the ratio of signal to background events is at the heart of searches for new particles. We use 


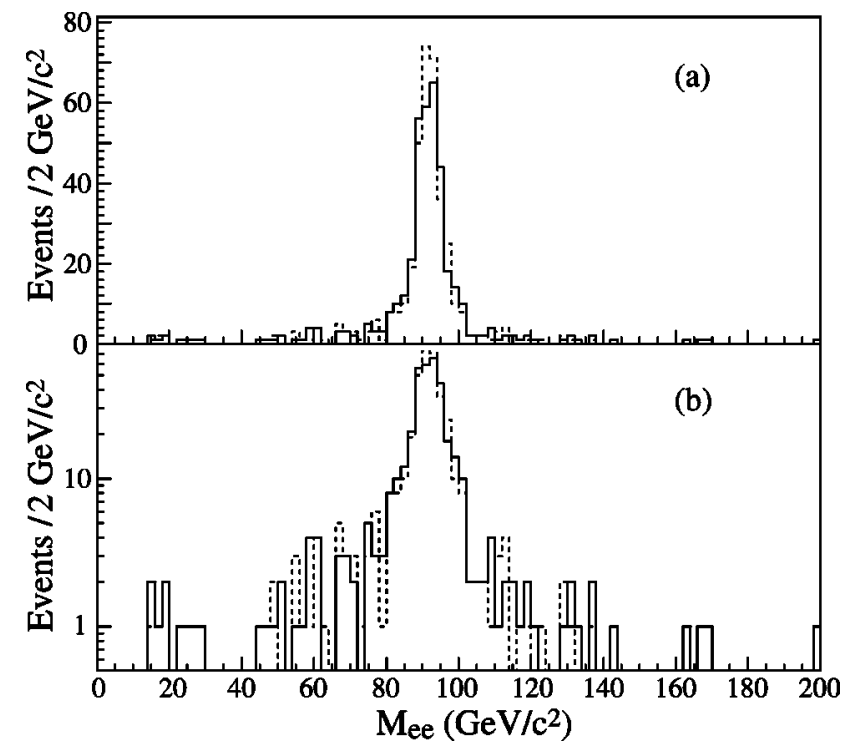

FIG. 1. $Z(\rightarrow e e)+2 j$ data before (solid) and after (dashed) revertexing: (a) has a linear scale and illustrates the improvement in the $Z$-boson mass resolution after the revertexing; (b) has a logarithmic scale and shows the suppression of the background from vertex misidentification in the tails of the $Z$-boson peak.

two optimization techniques to aid in this selection: the random grid search method, which has been used by $\mathrm{D} \emptyset$ in the measurement of the top-quark pair-production cross section [31] and in the search for the supersymmetric partner of the top quark [32], and neural network analysis, which has been used by $D \emptyset$ in the measurement of the top-quark mass $[33,27]$ and in the determination of the $t \bar{t}$-to-all-jets cross section $[34,35]$.

\section{A. Additional variables}

In addition to kinematic variables such as the transverse energies of electrons and jets and the $\boldsymbol{E}_{T}$ used in standard analyses, we study other variables to determine their efficiency in separating signal from background. These include the energy sums, event-shape variables, invariant-mass variables, and mass-difference variables listed below.

Energy and transverse energy sums:

$H_{T}^{e}$ is the sum of the $E_{T}$ of the two leptons [two electrons, or electron and neutrino $\left.\left(\boldsymbol{E}_{T}\right)\right]$;

$H_{T}^{j}$ is the sum of the $E_{T}$ of all jets;

$H_{T}^{j 12}$ is the sum of the $E_{T}$ of the two leading jets;

$H_{T}^{j 123}$ is the sum of the $E_{T}$ of the three leading jets;

$S_{T}=H_{T}^{e}+H_{T}^{j}$

$S_{T}^{12}=H_{T}^{e}+H_{T}^{j 12}$; and

$S$ is the total energy in the event.

Event-shape variables:

centrality $\left(S_{T} / S\right)$;

aplanarity of jets and leptons $[27,36]$;

sphericity [36]; and

the rms of the $E_{T}$-weighted distribution in jet $\eta$ [34].

Invariant-mass variables:

$M_{e e}$ is the dielectron invariant mass;

$M_{e j}$ is the invariant mass of various electron and jet combinations; and
$M_{T}^{e \nu}$ is the electron-neutrino transverse mass.

Mass-difference variables for the $e e j j$ analysis:

$$
\begin{aligned}
\frac{\delta M}{M}\left(M_{\mathrm{LQ}}\right) & =\frac{\sqrt{\left(M_{\mathrm{LQ}_{1}}-M_{\mathrm{LQ}}\right)^{2}+\left(M_{\mathrm{LQ}_{2}}-M_{\mathrm{LQ}}\right)^{2}}}{M_{\mathrm{LQ}}}, \\
\frac{\delta M}{M} & =\frac{M_{\mathrm{LQ}_{1}}-M_{\mathrm{LQ}_{2}}}{\left(M_{\mathrm{LQ}_{1}}+M_{\mathrm{LQ}_{2}}\right) / 2}, \\
\frac{\delta M}{\sqrt{M}} & =\frac{M_{\mathrm{LQ}_{1}}-M_{\mathrm{LQ}_{2}}}{\sqrt{\left(M_{\mathrm{LQ}_{1}}+M_{\mathrm{LQ}_{2}}\right) / 2}},
\end{aligned}
$$

where $M_{\mathrm{LQ}_{1}}$ and $M_{\mathrm{LQ}_{2}}$ are the electron-jet invariant-mass combinations that are closest to each other, and $M_{\mathrm{LQ}}$ is the hypothesized leptoquark mass.

Mass-difference variable for the $e \nu j j$ analysis:

$$
\frac{\delta M}{M}\left(M_{\mathrm{LQ}}\right)=\min \left(\frac{\left|M_{e j 1}-M_{\mathrm{LQ}}\right|}{M_{\mathrm{LQ}}}, \frac{\left|M_{e j 2}-M_{\mathrm{LQ}}\right|}{M_{\mathrm{LQ}}}\right),
$$

where $M_{e j 1}$ and $M_{e j 2}$ are the invariant masses of the electron with the first jet and the second jet, respectively, and $M_{\mathrm{LQ}}$ is the hypothesized leptoquark mass.

Over 50 combinations of these variables were used in the random grid search and neural network studies described below to determine the optimal set of variables and selection criteria for the $e e j j$ and $e \nu j j$ channels.

\section{B. Optimization criterion}

If first-generation leptoquarks with a mass of approximately $200 \mathrm{GeV} / \mathrm{c}^{2}$ exist, we want to achieve the highestpossible discovery significance. If there is no evidence of leptoquark production, we want to set the lowest possible 95\% C.L. limit on their production cross section. Based on the Monte Carlo (MC) simulations of the signal and the background estimates described below, we pursue a fixedbackground strategy for our search. We optimize our selection criteria by maximizing the signal efficiency for 0.4 expected background events. This method leads to excellent discovery potential and a $67 \%$ probability that no background events will be observed. If no events are observed, the experimental limit has the advantage of being independent of the predicted number of background events and its uncertainty.

\section{Random grid search}

The random grid search method, which was implemented as the computer program RGSEARCH [37], helps determine the set of cuts that optimally separates signal from background. In a standard grid search, the signal and background acceptances for some cutoff $\left(x_{\text {cut }}\right)$ on a variable $x$ are determined for all values between some minimum and maximum, $x_{\min }$ and $x_{\max }$, respectively. A refinement of this technique is to use the MC signal to define the range of $x_{\text {cut }}$. For each MC event, $x_{\text {cut }}$ is set to the generated value of $x$, and the acceptances for signal and background are determined for that $x_{\text {cut }}$. While running RGSEARCH, the value of a cutoff on a variable can be fixed or allowed to vary in some range. Minimum and/or maximum values for $x_{\text {cut }}$ can be preset or, 
TABLE II. The level 2 triggers used in the $e e j j$ analysis. The runs listed correspond to different periods during Run 1 of the Tevatron (1992-1996). The transverse energy of an EM cluster is denoted by $E_{T}^{\mathrm{EM}}$. The number of events is that in the initial data set.

\begin{tabular}{lccc}
\hline \hline Run & Trigger requirements & Integrated luminosity & Number of events \\
\hline Run 1A & $E_{T}^{\mathrm{EM} 1, \mathrm{EM} 2}>10 \mathrm{GeV}$ & $14.7 \mathrm{pb}^{-1}$ & 1131 \\
Run 1B & $E_{T}^{\mathrm{EM} 1}>20 \mathrm{GeV}$, isolated & $97.8 \mathrm{pb}^{-1}$ & 7500 \\
& $E_{T}^{\mathrm{EM} 2}>16 \mathrm{GeV}$ & & 888 \\
Run 1C & $E_{T}^{\mathrm{EM} 1}>20 \mathrm{GeV}$, isolated & $10.5 \mathrm{pb}^{-1}$ & \\
& $E_{T}^{\mathrm{EM} 2}>16 \mathrm{GeV}$ & & \\
\hline \hline
\end{tabular}

alternatively, any values that are allowed for signal can be used in the search. In general, the search is multidimensional, and many combinations of variables, both fixed and varying, are studied to find an optimal set of requirements to impose on the data. Trigger thresholds and other criteria used to define the initial data sample are also imposed in all RGSEARCH trials. One of the results of an RGSEARCH trial is a plot of the number of expected signal events versus the predicted number of background events, normalized to the luminosity of the data sample, including detection efficiencies.

\section{Neural network analysis}

We also use three-layer feed-forward neural networks $[38,39]$ in the search for leptoquarks. For each combination of $n$ variables, a network is trained using MC signal events $(S)$ and an appropriate mixture of background events $(B)$ to yield an output discriminant $\mathcal{D}_{\mathrm{NN}}$ near 1 for signal and 0 for background. For a sufficiently large sample of training events, when the trained network is applied to the data, the discriminant output from the neural network is approximately $S(x) /[S(x)+B(x)]$, where $S(x)$ and $B(x)$ are the $n$-variable signal and background densities. This defines contours of constant probability for signal versus background in the $n$-dimensional space that represent the optimal functions separating the signal from the background. The discriminant then becomes a single variable that can be used to optimize the analysis for any desired signal to background ratio.

\section{V. eejj CHANNEL}

The study of the eejj channel is particularly important because it is the only channel sensitive to leptoquarks with $\beta=1$. It is also sensitive to leptoquarks with $\beta<1$; however, since both leptoquarks have to decay in the charged-lepton mode, the cross section for leptoquark pair production and subsequent decay into the eejj channel is suppressed by a factor of $\beta^{2}$.

Independent of the scalar or vector nature of leptoquarks, the analyses are very similar. In particular the data sample and the final event selection are identical. We describe the scalar leptoquark analysis first, in detail, and then the vector leptoquark analysis.

\section{A. Data}

\section{Triggers}

Events with two electrons satisfying the online trigger requirements listed in Table II are used as the starting sample for the dielectron data sample. The total integrated luminosity for these triggers is $123.0 \pm 7.0 \mathrm{pb}^{-1}$, which corresponds to sample of 9519 events. The average trigger efficiency for the data in this analysis is $(99.5 \pm 0.5) \%$.

\section{Event selection for the base data sample}

We require two electrons with $E_{T}^{e}>20 \mathrm{GeV}$ and at least two jets with $E_{T}^{j}>15 \mathrm{GeV}$. As described in Sec. III, only one of the electrons is required to have a matching track. Events containing an electron close to a jet $\left(\Delta \mathcal{R}_{e}<0.7\right)$ are rejected. Events whose dielectron invariant mass lies inside the $Z$-boson mass window, $82<M_{e e}<100 \mathrm{GeV} / c^{2}$, are also removed. After identification, fiducial, initial kinematic, and $M_{e e}$ requirements, 101 events remain. We call these events the base data sample.

\section{B. MC signal samples}

Leptoquark pair production in the eejj channel can be modeled as the production of a pair of identical stronglyinteracting particles, each of which decays into an electron and a jet. Monte Carlo events simulating the pair production of scalar leptoquarks are generated using ISAJET [40] for leptoquark masses from 80 to $250 \mathrm{GeV} / \mathrm{c}^{2}$. The ISAJET samples are used only for calculating acceptances; the NLO calculation of Ref. [22] is used for the production cross section.

\section{Background samples}

The primary backgrounds to the $e e j j$ final state are from $e^{+} e^{-}$("Drell-Yan") production with two or more jets, $t \bar{t}$ production, and multijet events in which two jets are misidentified as electrons.

\section{Drell-Yan background}

Drell-Yan (DY) events are generated using ISAJET in four mass ranges: $20-60,60-120,120-250$, and 250-500 GeV/ $c^{2}$. For calculating the background, the DY $+2 j$ cross section from ISAJET is normalized to the observed number of events in the Z-boson mass peak after imposition of the kinematic criteria described above. The scaling factor is 1.7 \pm 0.1 and reflects the fact that ISAJET does not provide the NLO corrections ("K-factor") to the LO DY production cross section. The uncertainty in this background is $20 \%$, dominated by the $15 \%$ uncertainty in the jet energy scale. We estimate that the base data sample contains 66.8 \pm 13.4 DY events. 


\section{2. $t \bar{t}$ Background}

The $t \bar{t} \rightarrow$ dileptons MC sample is produced using HERWIG [41] for $m_{t}=170 \mathrm{GeV} / c^{2}$. The events are representative of all $e e, e \mu$ and $\mu \mu$ final states, including those from $\tau$ decay. The sample of 101339 events corresponds to an integrated luminosity of about $270 \mathrm{fb}^{-1}$. The D $\emptyset$ measurement [31] of the $t \bar{t}$ production cross section has an uncertainty of $35 \%$. This, when combined with the $15 \%$ uncertainty in the jet energy scale, leads to an overall uncertainty of $38 \%$ in the predicted number of $t \bar{t}$ events. The base data sample is estimated to contain $1.8 \pm 0.7 t \bar{t}$ events.

\section{Photon background}

Direct photon production is the main source of real photons (observed as EM objects without associated tracks) in the $e e j j$ final state; its contribution is small and is taken into account when the multijet background is estimated. Other sources of photons, such as $W \gamma+2 j$ production, are negligible for high- $E_{T}$ photons.

\section{Multijet background}

The multijet background is estimated using data collected with a trigger that required three jets with $E_{T}^{j}>10 \mathrm{GeV}$ at level 2. This trigger was prescaled and had an integrated luminosity of $0.936 \mathrm{pb}^{-1}$. Two sets of events are selected from this trigger. Events in the $3 j$ sample are required to have at least two jets with $E_{T}^{j}>15 \mathrm{GeV}$ and at least one additional jet with $E_{T}^{j}>20 \mathrm{GeV}$. Events in the $2 j+\mathrm{EM}$ sample have an EM object with $E_{T}^{\mathrm{EM}}>20 \mathrm{GeV}$ rather than a third jet.

The probabilities for a jet to be misidentified as either an electron or trackless electron are determined by comparing the number of candidates with $E_{T}^{e}>E_{0}$ that pass standard quality cuts in the $2 j+$ EM sample and the total number of jets with $E_{T}^{j}>E_{0}$ in the $3 j$ sample. The $E_{0}$ threshold is varied from 20 to $50 \mathrm{GeV}$, and the probabilities are stable for a cut value above $25 \mathrm{GeV}$, i.e., above the jet trigger turn-on. The probabilities for a jet to be misidentified as an electron with a track and without a track are measured to be

$$
\begin{gathered}
f_{\text {track }}=(3.50 \pm 0.35) \times 10^{-4}, \\
f_{\text {no track }}=(1.25 \pm 0.13) \times 10^{-3},
\end{gathered}
$$

and, within the uncertainties, are independent of the $E_{T}$ and pseudorapidity of the electron. These values are crosschecked using the ratio of $3 j+\mathrm{EM}$ and $4 j$ events. This method of determining the misidentification probability automatically accounts for the direct photon background that is a part of the general "multijet" background.

We then apply these misidentification probabilities to the weighted number of $4 j$ events in the $3 j$ sample. The weight assigned to each event is the number of jet permutations that can be used to misidentify a pair of EM objects. The backgrounds in the two samples, two electrons or an electron and a trackless electron, are estimated by multiplying the weighted number of events by $f_{\text {track }}^{2}$ or $2 f_{\text {track }} f_{\text {no track }}$, respec- tively. We assign an uncertainty of $15 \%$ to these values, which reflects the variation of the misidentification probabilities as a function of $E_{T}^{e}$, any difference between the $\mathrm{CC}$ and $\mathrm{EC}$, as well as certain jet trigger turn-on effects. The number of misidentified multijet events in the base data sample is estimated to be $24.3 \pm 3.6$ events.

\section{Total background}

The total background estimate for the base data sample is $92.8 \pm 13.8$ events, in agreement with the 101 events observed in the data.

\section{Electron identification efficiencies}

There are approximately $300 \mathrm{Z}$-boson events remaining in the initial data sample after all requirements except those on the dielectron mass and for electron identification. This is sufficient to estimate the identification efficiencies for $\mathrm{CC}$ $\mathrm{CC}, \mathrm{CC}-\mathrm{EC}$, and EC-EC electron combinations.

We plot the dielectron mass spectrum without any electron identification requirements beyond EM object reconstruction and subtract the multijet and DY backgrounds using the standard "side-band" technique. We then apply the electron identification requirements, again subtracting the backgrounds using the same side-band technique. The ratio of the background-subtracted number of $Z$ bosons with the identification requirements to that without the identification requirements gives the efficiency per event. The efficiency is $(74 \pm 3) \%,(66 \pm 4) \%$, and $(68 \pm 9) \%$ for CC-CC, CC-EC, and EC-EC electron combinations, respectively.

To calculate the average efficiency for leptoquark events, we find the relative fractions of the CC-CC, CC-EC, and EC-EC topologies. These are the same, within the errors, for leptoquark masses of 180,200 , and $220 \mathrm{GeV} / \mathrm{c}^{2}$, and equal $(83 \pm 2) \%,(16 \pm 1) \%$, and $(1.1 \pm 0.2) \%$ for CC-CC, CCEC, EC-EC combinations, respectively. These fractions and the electron identification efficiencies give an overall electron identification efficiency of $(73 \pm 4) \%$ for leptoquark masses between 180 and $220 \mathrm{GeV} / c^{2}$.

\section{E. Event selection optimization}

\section{Random grid search}

Extensive testing of combinations of the variables described in Sec. IV A shows that the use of a single variable, the scalar sum of the transverse energies of all the objects in the event, $S_{T}$, is the most powerful. Figure 2 shows the $S_{T}$ distribution for the base data sample, the predicted background, and a sample of $200 \mathrm{GeV} / c^{2}$ leptoquark MC events. All of the leptoquark MC samples and the DY, $t \bar{t}$, and $2 j$ + EM background samples are used in the random grid search. The leptoquark events are used to set the trial threshold values for the different parameters. The number of predicted background events is determined using the three background samples. Shown in Fig. 3 is the predicted number of signal events versus the expected number of background events for three different RGSEARCH trials, where the samples have been normalized to an integrated luminosity of 123 


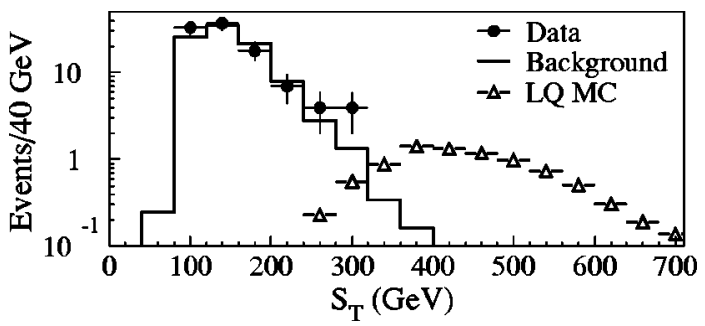

FIG. 2. $S_{T}$ distributions for background (solid line histogram), data (solid circles), and $M_{\mathrm{LQ}}=200 \mathrm{GeV} / \mathrm{c}^{2} \mathrm{MC}$ events (open triangles) for the $e e j j$ analysis.

$\mathrm{pb}^{-1}$, and the detection efficiencies, as well as the kinematic acceptance for the RGSEARCH thresholds, have been included. In these trials, the $E_{T}$ thresholds of the two electrons and the two jets are fixed to those in the base data sample. The thresholds varied are those for $S_{T}$ alone, for $\frac{\delta M}{M}(200)$ alone, and for these two variables together. When combined, $S_{T}$ and the mass-difference variable yield a higher signal efficiency for very low values of expected background (less than 0.3 events), but the result is comparable to the use of $S_{T}$ alone when the expected background is approximately 0.4 events. For the same expected background, using just the mass-difference variable leads to a $10 \%$ reduction in the predicted number of signal events compared to that using just $S_{T}$. Requiring $S_{T}>350 \mathrm{GeV}$ leads to approximately $0.4 \mathrm{ex}-$ pected background events (see Sec. IV B). The highest value of $S_{T}$ seen in the data is $312 \mathrm{GeV}$; therefore, no events pass this requirement.

\section{Neural network analysis}

The analysis based on the random grid search uses the linear sum $S_{T} \equiv H_{T}^{e}+H_{T}^{j}$. However, it is possible that a func-

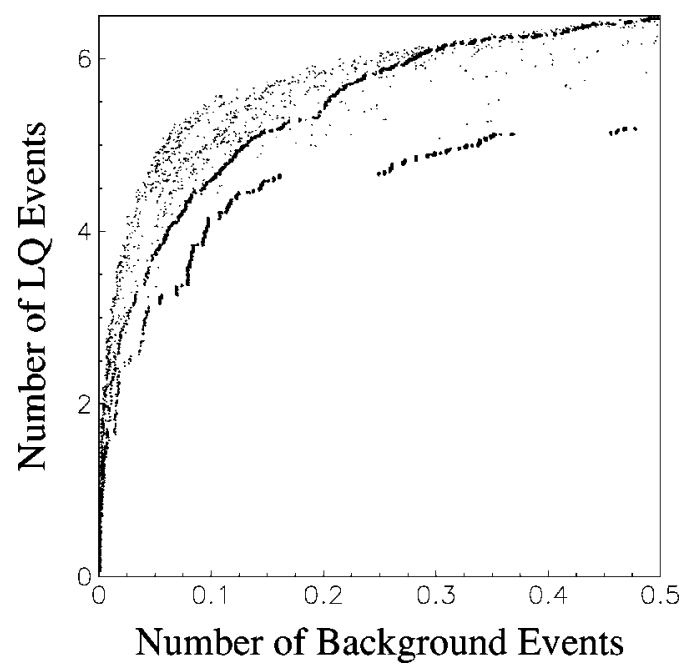

FIG. 3. Predicted number of $M_{\mathrm{LQ}}=200 \mathrm{GeV} / c^{2}$ events vs the predicted number of background events for three RGSEARCH runs. The upper dotted line shows the variation with $S_{T}$. The lower dotted line shows the variation with $(\delta M / M)(200)$. The structure (gaps) arises from an increase in acceptance for DY events. The more dispersed set of dots shows the result when both $S_{T}$ and $(\delta M / M)(200)$ are varied. The density of the points is irrelevant.

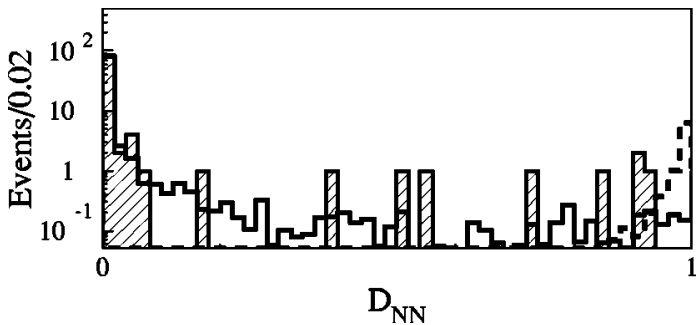

FIG. 4. Comparison of $\mathcal{D}_{\mathrm{NN}}$ distributions for the predicted background (solid line histogram), $200 \mathrm{GeV} / c^{2}$ leptoquark events (dashed line histogram), and the data (hatched histogram).

tion other than a simple linear sum is the optimal way to combine the two variables. The simplest way to compute this function is with a two-dimensional neural network. For this approach, we use a neural network with two input nodes (corresponding to the variables $H_{T}^{e}$ and $H_{T}^{j}$ ), three hidden nodes, and one output node. The network is trained using the $200 \mathrm{GeV} / \mathrm{c}^{2}$ leptoquark MC sample as signal (with a desired network output $\mathcal{D}_{\mathrm{NN}}=1$ ) and the observed admixture of DY, $t \bar{t}$, and multijet events as background (with desired $\mathcal{D}_{\mathrm{NN}}$ $=0$ ). Figure 4 shows the distribution of $\mathcal{D}_{\mathrm{NN}}$ for the background, the $200 \mathrm{GeV} / \mathrm{c}^{2}$ leptoquark MC events, and the data. The discrimination between signal and background is good.

Each value of $\mathcal{D}_{\mathrm{NN}}$ defines a contour of constant probability between signal and background in the $\left(H_{T}^{e}, H_{T}^{j}\right)$ plane. The expected distributions in $x \equiv\left(H_{T}^{e}, H_{T}^{j}\right)$ space for a 200 $\mathrm{GeV} / \mathrm{c}^{2}$ leptoquark signal, the background, and the data are shown in Fig. 5. The contours corresponding to $\mathcal{D}_{\mathrm{NN}}=0.5$, 0.8 , and 0.95 are also shown.

Selecting events with $\mathcal{D}_{\mathrm{NN}}>0.95$ yields approximately 0.4 background events. The highest value of $\mathcal{D}_{\mathrm{NN}}$ in the data
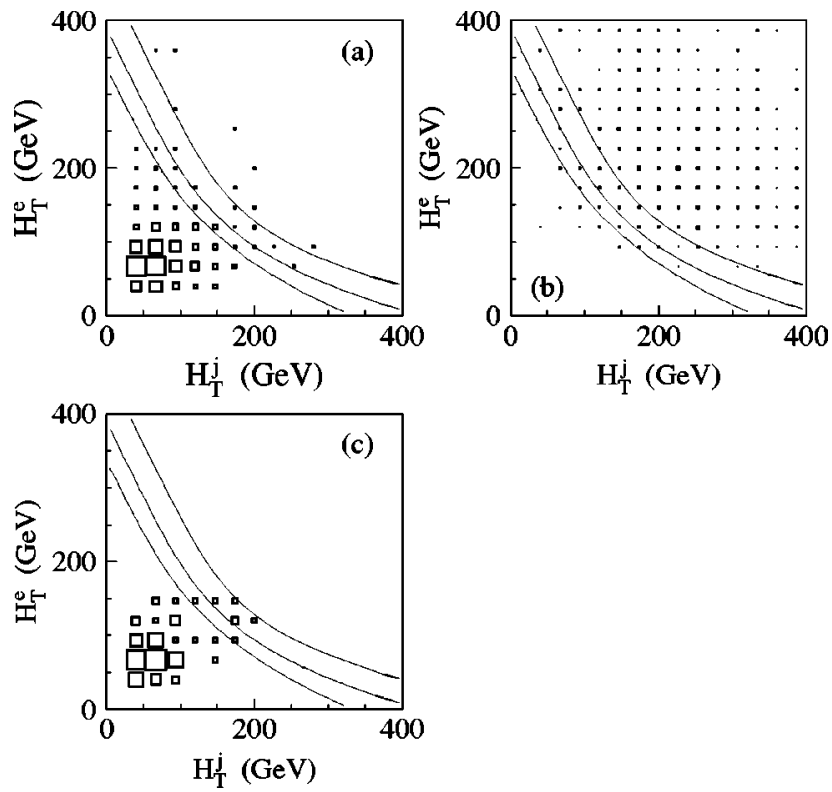

FIG. 5. $H_{T}^{e}$ vs $H_{T}^{j}$ for (a) the predicted background, (b) 200 $\mathrm{GeV} / \mathrm{c}^{2}$ leptoquark events, and (c) the base data sample. The curved lines correspond to $\mathcal{D}_{\mathrm{NN}}=0.5,0.8$, and 0.95 (from left to right). The area of a displayed square is proportional to the number of events in that bin, with the total number of events normalized to $123 \mathrm{pb}^{-1}$. 


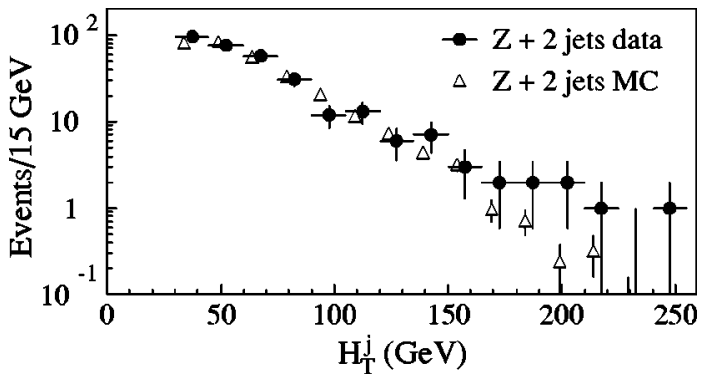

FIG. 6. The $H_{T}^{j}$ distribution for $Z+2 j$ data (solid circles) and $\mathrm{MC}$ (open triangles) in the $Z$-boson mass region. For high-mass DY events, $S_{T} \approx 350 \mathrm{GeV}$ corresponds to $H_{T}^{j} \approx 100 \mathrm{GeV}$.

is 0.92 and no events survive the selection. The efficiency for identifying $200 \mathrm{GeV} / \mathrm{c}^{2}$ leptoquark events using the neural network analysis is nearly identical to the efficiency found using the $S_{T}$ analysis. Since the two methods give essentially equivalent results for the final experimental limits, we use the simpler $S_{T}$ analysis based on the random grid search described in Sec. V E.

\section{F. Checks}

\section{1. $S_{T}$ distribution}

The modeling of the $S_{T}$ distribution for high-mass DY events is checked by studying $H_{T}^{e}$ and $H_{T}^{j}$ separately, using data and $\mathrm{MC}$ events in the $Z$-boson mass region. The average value of $H_{T}^{e}$ for high-mass DY events (which provide most of the DY background) is approximately $250 \mathrm{GeV}$, corresponding to an $H_{T}^{j}$ of approximately $100 \mathrm{GeV}$ for $S_{T}=350 \mathrm{GeV}$. The distribution of $H_{T}^{j}$ for high-mass DY events is expected to be similar to that for $Z+2 j$ events. Figure 6 shows the $H_{T}^{j}$ distribution for $Z+2 j \mathrm{MC}$ and data. In the region corresponding to the $S_{T}$ cutoff for high-mass DY events $\left(H_{T}^{j}\right.$ $\approx 100 \mathrm{GeV}$ ), the agreement is good. Disagreement between the $Z+2 j \mathrm{MC}$ events and the data at higher values of $S_{T}$ stems from the LO calculations used in the simulation and does not affect the results of this analysis.

In addition, we fit the $H_{T}^{j}$ distribution of the data to a sum of the DY and multijet backgrounds (the expected $t \bar{t}$ background is smaller than the uncertainties in the fit and is neglected). Figure 7 shows the $H_{T}^{j}$ distribution for the data and the result of the fit for the two backgrounds. The fit yields $77.5 \pm 15.9$ DY events and $24.6 \pm 13.9$ misidentified multijet events, for a total of $102 \pm 21$ events, in agreement with the 101 events in the base data sample and with the direct determination of the two dominant background contributions.

\section{Mass fitting}

To improve resolution, rather than simply calculating the invariant masses of the electron-jet pairs, we use a kinematic fitter to reconstruct the mass of two identical particles that decay to electron + jet. The $\mathrm{D} \emptyset$ fitting package KFIT is based on the bubble-chamber fitting program SQUAW [42].

The fitter balances the two electrons and the two leading jets against any extra jets and unclustered energy in the event

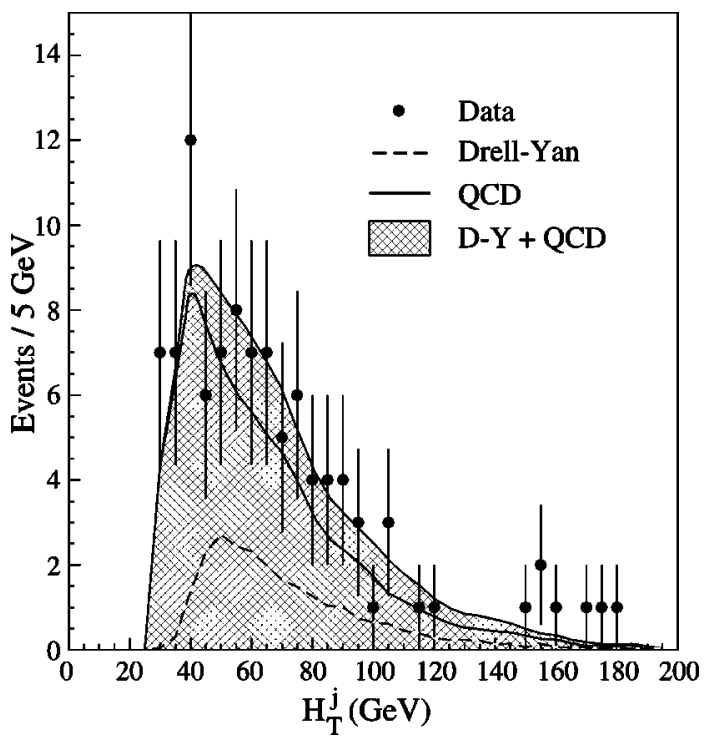

FIG. 7. Fit of the $H_{T}^{j}$ distribution in the $e e j j$ data to the sum of the DY and multijet backgrounds.

by minimizing a $\chi^{2}$ to find the best fit solution. The $\chi^{2}$ takes into account the object resolutions (see Sec. III) as well as the kinematic constraints. Three constraints are used in the fit: momentum conservation in the $x$ and $y$ directions for electrons, jets and unclustered energy, and the equivalence in the mass of the two leptoquarks.

In each event there are two ways to associate the electrons and two leading jets $\left(e_{1} j_{1}, e_{2} j_{2}\right.$ and $\left.e_{1} j_{2}, e_{2} j_{1}\right)$. Fits for both configurations are performed and the configuration with the lowest $\chi^{2}$ is retained. The mass distribution for the background is found using the MC samples for DY and $t \bar{t}$ events; the multijet sample is not large enough to parametrize a smooth line shape, so a jet is used to simulate an electron in the fit.

Figure 8 shows $S_{T}$ as a function of the fitted mass for the background, the $200 \mathrm{GeV} / \mathrm{c}^{2}$ leptoquark MC sample, and the data, before the $S_{T}>350 \mathrm{GeV}$ requirement. The background is centered at low $S_{T}$ and low fitted mass and does not resemble the leptoquark signal. The data most closely resemble the expected background. Figure 9 displays the onedimensional distributions in fitted mass for the three samples before the $S_{T}$ cut and with a reduced $S_{T}>250 \mathrm{GeV}$ requirement. The data and the predicted background are in good agreement.

\section{Varying the $S_{T}$ threshold}

Table III shows a comparison between the predicted number of events from each of the three background sources, the total background, and the number of events observed in the data as a function of $S_{T}$ threshold. The agreement between the predicted background and the data is excellent.

\section{G. Signal studies}

\section{Systematic uncertainties}

The systematic uncertainties in the signal acceptance are obtained by comparing the results for scalar leptoquark 

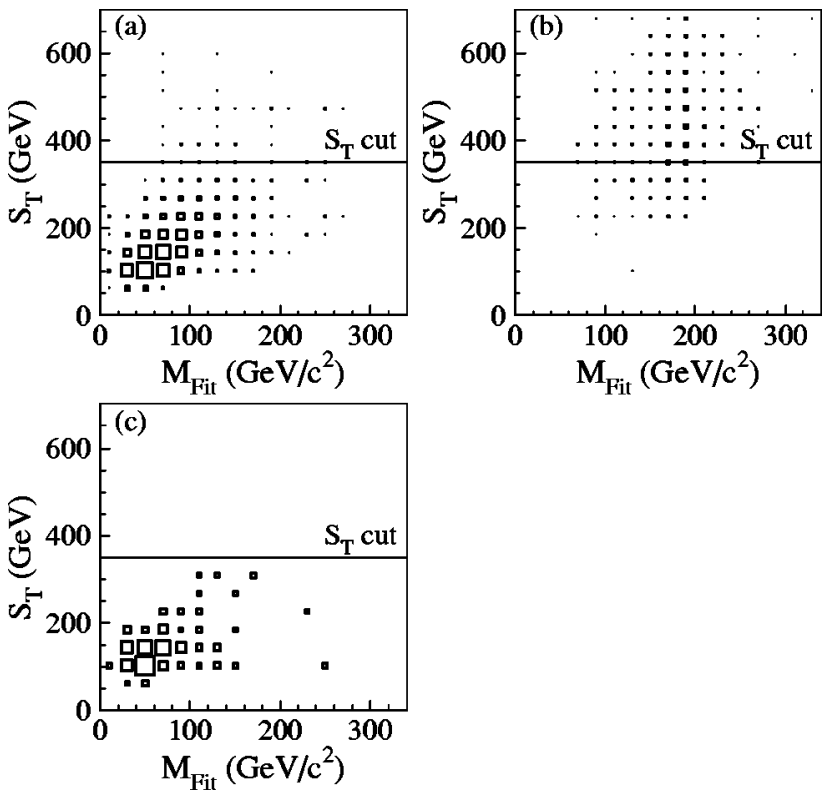

FIG. 8. $S_{T}$ vs the fitted mass for (a) background, (b) $200 \mathrm{GeV} / \mathrm{c}^{2}$ leptoquarks, and (c) the base data sample. The area of a displayed square is proportional to the number of events in the bin.

samples generated using ISAJET and PYTHIA with different structure functions and renormalization scales. The uncertainty from the jet energy scale is determined by varying the calorimeter response to jets by one standard deviation. The systematic error in the signal varies from $17 \%$ to $13 \%$ for leptoquark masses between 120 and $250 \mathrm{GeV} / \mathrm{c}^{2}$. The systematic uncertainties are summarized in Table IV.

\section{Signal efficiency}

The signal-detection efficiencies are determined using simulated scalar leptoquark events that pass the selection requirements and are shown in Table V. The uncertainties in the efficiencies include uncertainties in trigger and particle identification, the jet energy scale, effects of gluon radiation and parton fragmentation in the modeling, and finite Monte Carlo statistics. The overall efficiency ranges from $1 \%$ to $38.5 \%$ for leptoquark masses between 80 and $250 \mathrm{GeV} / \mathrm{c}^{2}$.
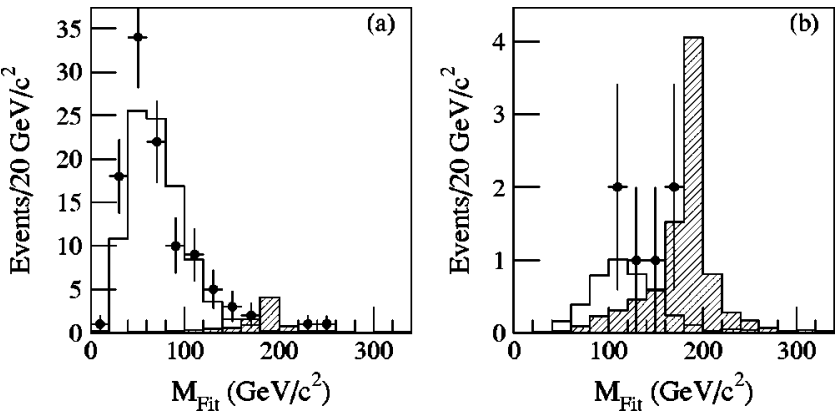

FIG. 9. Distributions of the fitted mass for events in the base data sample (solid circles), expected background (solid line histogram), and $200 \mathrm{GeV} / \mathrm{c}^{2}$ leptoquarks (hatched histogram) with (a) no cut on $S_{T}$ and (b) a reduced threshold of $S_{T}>250 \mathrm{GeV}$.
TABLE III. Comparison of the number of events expected from the background with the number observed for the $e e j j$ analysis as a function of the threshold on $S_{T}$.

\begin{tabular}{cccccr}
\hline \hline $\begin{array}{c}S_{T} \text { threshold } \\
(\mathrm{GeV})\end{array}$ & DY & Multijet & $t \bar{t}$ & Total background & Data \\
\hline 0 & 66.8 & 24.3 & 1.79 & $92.8 \pm 13.8$ & 101 \\
100 & 61.0 & 23.2 & 1.79 & $85.9 \pm 12.7$ & 85 \\
125 & 45.0 & 16.9 & 1.75 & $63.7 \pm 9.36$ & 63 \\
150 & 28.8 & 10.2 & 1.65 & $40.6 \pm 5.96$ & 39 \\
175 & 16.0 & 5.67 & 1.44 & $23.1 \pm 3.32$ & 20 \\
200 & 9.12 & 3.16 & 1.15 & $13.4 \pm 1.93$ & 15 \\
225 & 4.88 & 1.73 & 0.84 & $7.45 \pm 1.06$ & 9 \\
250 & 2.64 & 0.99 & 0.59 & $4.22 \pm 0.59$ & 8 \\
275 & 1.35 & 0.60 & 0.39 & $2.34 \pm 0.32$ & 5 \\
300 & 0.75 & 0.35 & 0.25 & $1.35 \pm 0.19$ & 3 \\
325 & 0.31 & 0.23 & 0.16 & $0.70 \pm 0.09$ & 0 \\
350 & 0.18 & 0.16 & 0.11 & $0.44 \pm 0.06$ & 0 \\
375 & 0.12 & 0.11 & 0.07 & $0.30 \pm 0.04$ & 0 \\
400 & 0.07 & 0.08 & 0.04 & $0.20 \pm 0.03$ & 0 \\
\hline \hline
\end{tabular}

\section{H. Results from the eeejj channel for scalar leptoquarks}

Based on our observation of no events after requiring $S_{T}$ $>350 \mathrm{GeV}$, we set a $95 \%$ C.L. upper limit on the leptoquark pair-production cross section using a Bayesian approach [43] with a flat prior distribution for the signal cross section. Limits for different leptoquark masses are summarized in Table V. As indicated before, to compare our experimental results with theory, we use the NLO calculation of the production cross section [22]. This cross section is tabulated for a wide range of leptoquark masses and has the value of $0.184_{-0.026}^{+0.018} \mathrm{pb}$ for a $200-\mathrm{GeV} / \mathrm{c}^{2}$ leptoquark. The theoretical uncertainty corresponds to the variation of the renormalization scale $\mu$ used in the calculation from $2 M_{\mathrm{LQ}}$ to $\frac{1}{2} M_{\mathrm{LQ}}$. To set a limit on the leptoquark mass, we compare the theoretical cross section for $\mu=2 M_{\mathrm{LQ}}$ with our experimental limit, resulting in $M_{\mathrm{LQ}}>225 \mathrm{GeV} / c^{2}$ for a scalar leptoquark with $\beta=1$ and $M_{\mathrm{LQ}}>176 \mathrm{GeV} / c^{2}$ for a scalar leptoquark with $\beta=\frac{1}{2}$. Figure 10 shows the experimental limit as a function of scalar leptoquark mass along with the predicted cross sections for $\beta=1$ and $\beta=\frac{1}{2}$. The Collider Detector at Fermilab

TABLE IV. Systematic uncertainties in the signal for the $e e j j$ analysis.

\begin{tabular}{lc}
\hline \hline Source & Uncertainty $(\%)$ \\
\hline Particle identification & 5 \\
Smearing in the detector & 3 \\
Jet energy scale & $11-2\left(M_{\mathrm{LQ}}=120-250 \mathrm{GeV} / c^{2}\right)$ \\
Gluon radiation & 7 \\
PDF and $Q^{2}$ scale & 7 \\
Monte Carlo statistics & 2 \\
Luminosity & 5 \\
\hline Total & $17-13\left(M_{\mathrm{LQ}}=120-250 \mathrm{GeV} / c^{2}\right)$ \\
\hline \hline
\end{tabular}


TABLE V. Efficiency, background, 95\% C.L. upper limit on the leptoquark pair production cross section $\left(\sigma_{\text {limit }}\right)$, and the NLO cross section $\left(\sigma_{\mathrm{NLO}}\right)$ with $\mu=2 M_{\mathrm{LQ}}$ [22] for $\beta=1$ as a function of leptoquark mass for the eejj channel.

\begin{tabular}{rcccc}
\hline \hline $\begin{array}{c}\text { Mass } \\
\left(\mathrm{GeV} / c^{2}\right)\end{array}$ & $\begin{array}{c}\text { Efficiency } \\
(\%)\end{array}$ & $\begin{array}{c}\text { Background } \\
(\text { Events })\end{array}$ & $\begin{array}{c}\sigma_{\text {limit }} \\
(\mathrm{pb})\end{array}$ & $\begin{array}{c}\sigma_{\mathrm{NLO}} \\
(\mathrm{pb})\end{array}$ \\
\hline 80 & $1.0 \pm 0.2$ & $0.44 \pm 0.06$ & 2.9 & 36.0 \\
100 & $3.4 \pm 0.6$ & $0.44 \pm 0.06$ & 0.80 & 10.7 \\
120 & $8.8 \pm 1.4$ & $0.44 \pm 0.06$ & 0.30 & 3.81 \\
140 & $14.4 \pm 2.1$ & $0.44 \pm 0.06$ & 0.18 & 1.54 \\
160 & $20.9 \pm 3.0$ & $0.44 \pm 0.06$ & 0.13 & 0.68 \\
180 & $27.6 \pm 3.8$ & $0.44 \pm 0.06$ & 0.094 & 0.32 \\
200 & $33.2 \pm 4.0$ & $0.44 \pm 0.06$ & 0.076 & 0.16 \\
220 & $36.1 \pm 4.4$ & $0.44 \pm 0.06$ & 0.070 & 0.080 \\
225 & $37.7 \pm 4.5$ & $0.44 \pm 0.06$ & 0.067 & 0.068 \\
250 & $38.5 \pm 4.7$ & $0.44 \pm 0.06$ & 0.066 & 0.030 \\
\hline \hline
\end{tabular}

(CDF) Collaboration has set a lower limit of $M_{\mathrm{LQ}}>213$ $\mathrm{GeV} / c^{2}[17]$ for $\beta=1$. When our result is combined with the CDF limit, a Tevatron mass limit of $M_{\mathrm{LQ}}>242 \mathrm{GeV} / c^{2}$ is obtained for $\beta=1$ [44].

\section{Vector leptoquarks}

Vector leptoquark events were generated for leptoquark masses from 100 to $425 \mathrm{GeV} / \mathrm{c}^{2}$ using a version of PYTHIA [45] modified to include vector leptoquarks with various couplings. The distributions of the kinematic variables for scalar and vector leptoquarks are sufficiently similar that the same event selection can be used for both analyses.

The identification efficiencies for vector leptoquarks for the three couplings considered are identical within their un-

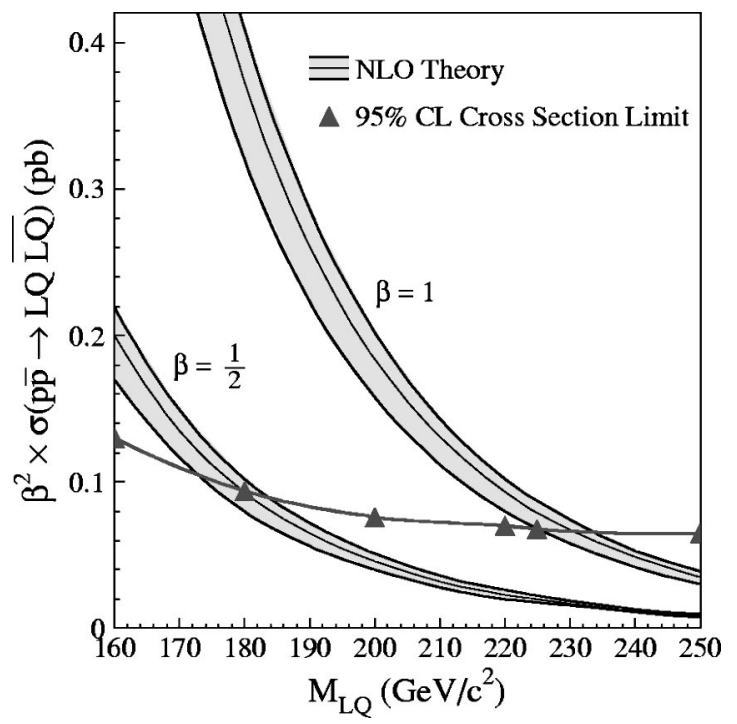

FIG. 10. Upper limit on the leptoquark pair-production cross section (triangles) from the eejj channel. The NLO calculations of Ref. [22] for $\beta=1$ (upper band) and $\beta=\frac{1}{2}$ (lower band) are also shown. The central lines correspond to $\mu=M_{\mathrm{LQ}}$, and the lower and upper edges of the bands correspond to $\mu=2 M_{\mathrm{LQ}}$ and $\mu$ $=\frac{1}{2} M_{\mathrm{LQ}}$, respectively.

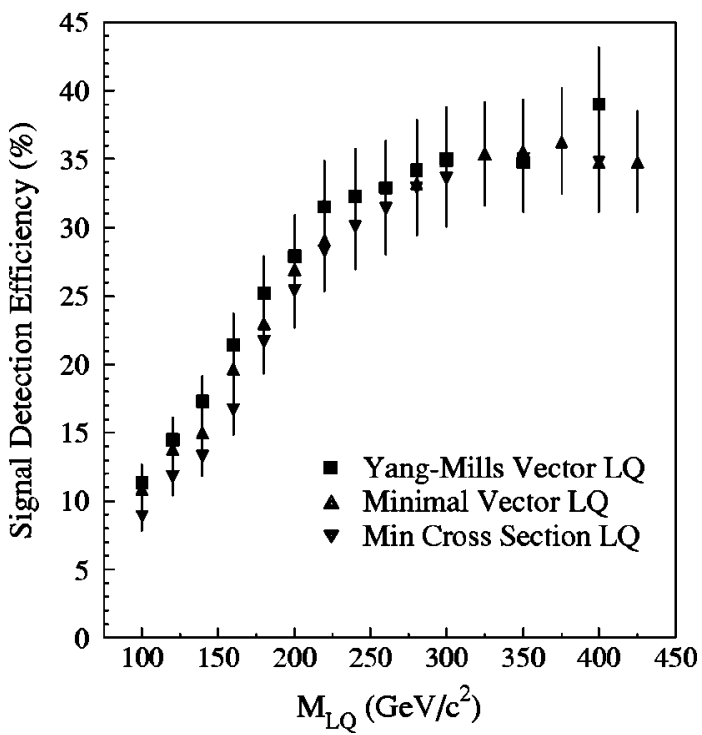

FIG. 11. The efficiency for identifying vector leptoquarks for the three couplings in the $e e j j$ channel. The differences between the efficiencies are small relative to the uncertainties.

certainties, as shown in Fig. 11. To reduce the statistical uncertainty from the $\mathrm{MC}$, we use the average identification efficiency of the three sets of $\mathrm{MC}$ events to set a single experimental limit on the cross section. This limit is then compared with the appropriate prediction for each coupling.

The cross sections for vector leptoquark production have been calculated only to LO for three gluon couplings [23]. For the scalar leptoquark case, cross sections calculated at NLO with $\mu=2 M_{\mathrm{LQ}}$ are approximately equal to those calculated at LO with $Q^{2}=M_{\mathrm{LQ}}^{2}$. We therefore compare our cross section limit with LO calculations of vector leptoquark cross sections for this choice of $Q^{2}$ scale.

Figure 12(a) shows the experimental limits along with the three theoretical vector leptoquark cross sections for the $e e j j$ channel for $\beta=1$. Here, the experimental result yields a lower limit of $M_{\mathrm{LQ}}>340 \mathrm{GeV} / c^{2}$ for the vector leptoquarks assuming Yang-Mills coupling, $M_{\mathrm{LQ}}>290 \mathrm{GeV} / c^{2}$ for minimal vector coupling, and $M_{\mathrm{LQ}}>245 \mathrm{GeV} / c^{2}$ for the coupling corresponding to the minimum cross section. Similarly, for $\beta=\frac{1}{2}$ [Fig. 12(b)], our result provides a lower limit of 300 $\mathrm{GeV} / c^{2}$ for Yang-Mills coupling, $250 \mathrm{GeV} / c^{2}$ for minimal vector coupling, and $210 \mathrm{GeV} / c^{2}$ for the coupling corresponding to the minimum cross section.

\section{VI. e vjj CHANNEL}

For $0<\beta<1$, leptoquark pairs can decay to $e \nu j j$ as well as to $e e j j$. The $e \nu j j$ channel therefore allows us to extend the leptoquark mass limit to higher masses for $0<\beta<1$. Our optimization techniques for this analysis are similar to those we used for the $e e j j$ channel.

As in the eejj channel, we use the same data sample for both the scalar and vector-leptoquark analyses. However, because the scalar-leptoquark analysis depends on a massbased variable, and the vector leptoquark analysis is sensitive to higher masses than the scalar leptoquark analysis, the final 


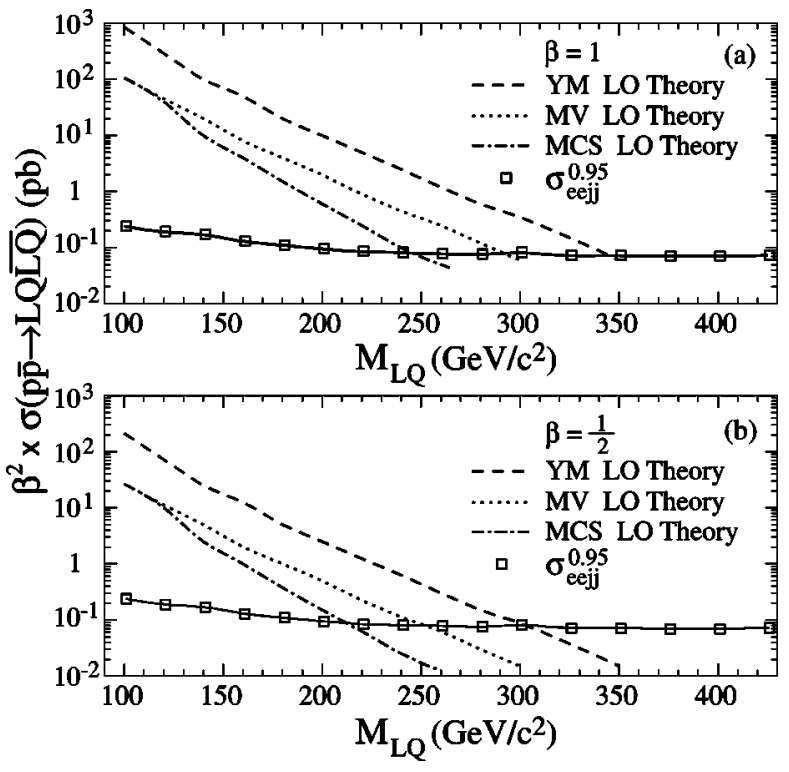

FIG. 12. The 95\% C.L. upper limits on the vector leptoquark pair production cross section from the eejj channel and the LO predictions for Yang-Mills (YM), minimal vector (MV), and minimum cross section (MCS) couplings as a function of leptoquark mass for (a) $\beta=1$ and (b) $\beta=\frac{1}{2}$.

event selection is slightly different. The scalar leptoquark analysis is described first, followed by the vector leptoquark analysis.

\section{A. Data}

\section{Triggers}

The data sample for this analysis corresponds to an integrated luminosity of $115 \pm 6 \mathrm{pb}^{-1}$. Using events collected with the triggers shown in Table VI, the initial data sample contains 95383 events.

\section{Event selection for the base data sample}

We require one electron with a matching track with $E_{T}^{e}$ $>30 \mathrm{GeV}, \boldsymbol{E}_{T}>20 \mathrm{GeV}$, and at least two jets with $E_{T}^{j}$ $>20 \mathrm{GeV}$. Electrons with $E_{T}^{e}>20 \mathrm{GeV}$ close to a jet $\left(\Delta \mathcal{R}_{e}<0.6\right)$ are "subtracted" from the jet in order not to double count the energy in the event. Since the $\boldsymbol{E}_{T}$ threshold
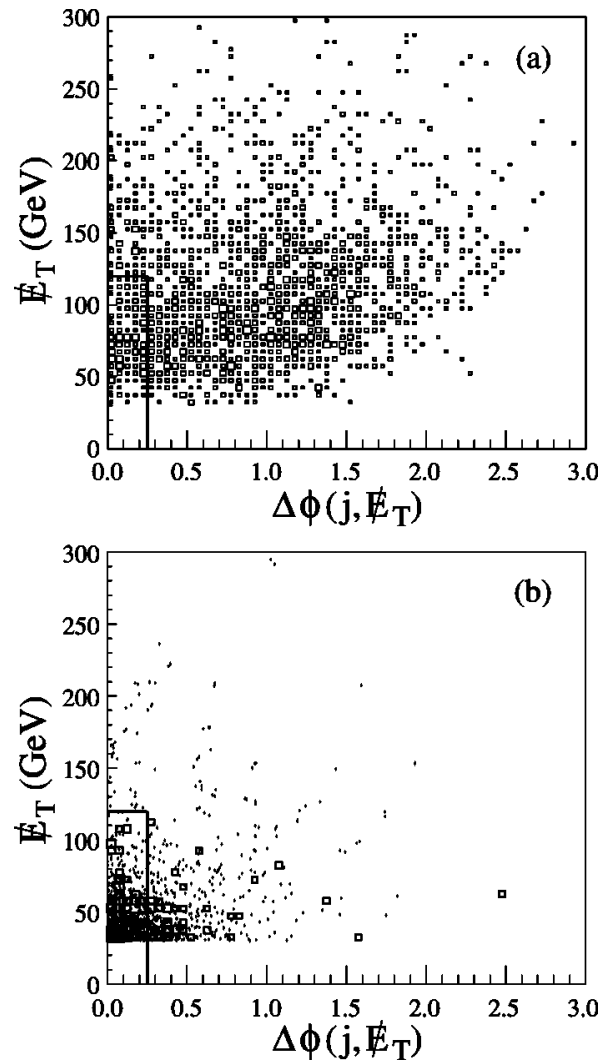

FIG. 13. Effect of the requirement of acolinearity in $\boldsymbol{E}_{T}$ on (a) a $180-\mathrm{GeV} / \mathrm{c}^{2} \mathrm{MC}$ leptoquark signal and (b) the multijet background. In (b), the dots show the distribution before imposition of the $M_{T}^{e \nu}$ requirement; the open squares show the distribution after applying the $M_{T}^{e v}$ requirement. The acolinearity requirement is indicated by the solid lines.

for this analysis is relatively high, we use a "minimal" Main Ring veto to increase the efficiency (see Sec. VI D 2).

To suppress the background from top-quark pair production, we apply a muon veto by requiring events to contain no well-reconstructed muons with $p_{T}>4 \mathrm{GeV} / c$ [26]. To reduce the multijet background when $\mathbb{E}_{T}<120 \mathrm{GeV}$, we require the $\boldsymbol{E}_{T}$ vector to be isolated in $\phi$ from any jets $\left(\Delta \phi\left(j, \boldsymbol{E}_{T}\right)>0.25\right)$. The effect of this requirement on a 180 $\mathrm{GeV} / \mathrm{c}^{2}$ leptoquark MC sample and on the multijet background is shown in Fig. 13.

After the above cuts, 1094 events remain in the data sample, primarily from $W+2 j$ production. To remove these

TABLE VI. The level 2 triggers used in the $e \nu j j$ analysis. The transverse energy of an EM cluster is denoted by $E_{T}^{\mathrm{EM}}$. The number of events is that in the initial data set.

\begin{tabular}{lccc}
\hline \hline Run & Trigger requirements & Integrated luminosity & Number of events \\
\hline Run 1A & $E_{T}^{\mathrm{EM}}>20 \mathrm{GeV}$ & $11.2 \mathrm{pb}^{-1}$ & 9862 \\
Run 1B & $E_{T}^{\mathrm{EM}}>20 \mathrm{GeV}$, isolated & $92.9 \mathrm{pb}^{-1}$ & 77912 \\
& $\boldsymbol{E}_{T}>15 \mathrm{GeV}$ & 369 \\
Run 1C & $E_{T}^{\mathrm{EM}}>20 \mathrm{GeV}$, isolated & $0.8 \mathrm{pb}^{-1}$ & 7240 \\
Run 1C & $\boldsymbol{E}_{T}>15 \mathrm{GeV}$ & $10.5 \mathrm{pb}^{-1}$ & \\
& $E_{T}^{\mathrm{EM}}>17 \mathrm{GeV}$, isolated \\
$E_{T}^{j_{1}, j_{2}}>10 \mathrm{GeV}, \boldsymbol{E}_{T}>14 \mathrm{GeV}$ & & \\
\hline \hline
\end{tabular}


events, we require $M_{T}^{e \nu}>110 \mathrm{GeV} / c^{2}$, reducing our base data sample to 14 events.

\section{B. MC signal samples}

We use the ISAJET event generator followed by the full detector simulation via GEANT to model the leptoquark signal. Two thousand to five thousand events were generated in steps of $20 \mathrm{GeV} / c^{2}$ for $M_{\mathrm{LQ}}$ between 80 and $220 \mathrm{GeV} / c^{2}$. We also use a PYTHIA MC sample at $200 \mathrm{GeV} / \mathrm{c}^{2}$ for studying MC systematics and for cross checks.

\section{Background samples}

As implied above, the dominant background to the $e \nu j j$ final state is $W+2 j$ production. The other significant backgrounds are from $t \bar{t}$ production and multijet events in which a jet is misidentified as an electron and the energy is mismeasured, thereby introducing false $\boldsymbol{E}_{T}$.

\section{1. $t \bar{t}$ background}

The $t \bar{t}$ MC event sample contains all leptonic final states for $m_{t}=170 \mathrm{GeV} / c^{2}$. It was generated using HERWIG followed by GEANT detector simulation. The sample corresponds to an integrated luminosity of about $32 \mathrm{fb}^{-1}$.

Since top-quark events frequently contain muons from $W \rightarrow \mu \nu$ and $b$-quark decays, the muon-veto requirement provides an effective way to remove $t \bar{t}$ events. To determine the background due to top quark events, we apply all of the basic cuts except the muon and minimal Main Ring vetoes to the MC sample.

Because the reconstruction efficiency for muons in MC events is higher than that for real muons, GEANT overestimates the rejection factor against muons. The correction (between $50 \%$ and $90 \%$ ) to the efficiency depends on the run number (due to chamber aging and repair) and the pseudorapidity of the muon. After applying this factor and the efficiencies described below, we estimate that the data sample of 1094 events (before imposing the $M_{T}^{e v}$ cut) contains $12 \pm 4 t \bar{t}$ events. After requiring $M_{T}^{e \nu}>110 \mathrm{GeV} / c^{2}, 2.0 \pm 0.7 t \bar{t}$ events are expected to remain in the base data sample of 14 events.

\section{Multijet background}

The multijet background is estimated using the data samples and the misidentification probability of (3.50 $\pm 0.35) \times 10^{-4}$ described in Sec. V C 4. We select events from the multijet data sample that have at least three jets and $\boldsymbol{E}_{T}>30 \mathrm{GeV}$. To minimize luminosity dependence and the misidentification of primary interaction vertices, we use only those events that have a single interaction vertex within the fiducial region of the detector $\left(\left|z_{\mathrm{VTX}}\right| \leqslant 50 \mathrm{~cm}\right)$. To account for multiple interactions and multiple vertices, we apply a correction factor. The correction factor is determined by measuring the fraction of single-interaction events in the $Z$ $+2 j$ data sample as a function of luminosity, and then weighting this fraction with a luminosity profile of the multijet data stream. The correction factor is $2.2 \pm 0.2$.
We next examine all three-jet combinations for each event. We treat each jet as an electron in turn and require each permutation to pass our electron and jet kinematic and fiducial requirements. Since the misidentification rate already accounts for the probability for a jet to be misidentified as an electron, we do not apply the electron identification criteria here. The multijet background is then defined by the product of the number of combinations that pass all criteria, the misidentification probability, and a factor that scales the multijet sample luminosity to the luminosity of the data. There are $75 \pm 15$ events expected in the sample of 1094 events before the $M_{T}^{e v}$ cut and $4.1 \pm 0.9$ multijet events after the $M_{T}^{e \nu}$ $>110 \mathrm{GeV} / \mathrm{c}^{2}$ requirement. The uncertainty in the background accounts for the statistics of the multijet sample and for a $20 \%$ systematic error reflecting the variation of the misidentification probability with $E_{T}$ and pseudorapidity, as well as jet trigger turn-on effects and the uncertainty in the scaling factor.

\section{3. $W+2 j$ background}

For the $W+2 j$ background, we use a sample of events generated with VECBOS [46] followed by ISAJET underlyingevent modeling and GEANT detector simulation. This initial sample contains 227726 events and corresponds to an integrated luminosity of approximately $0.8 \mathrm{fb}^{-1}$.

For calculating the background, the number of $\mathrm{MC} W$ $+2 j$ events with $M_{T}^{e v}<110 \mathrm{GeV} / c^{2}$ is normalized to the observed number of events after subtracting the estimated $t \bar{t}$ and multijet backgrounds. A scaling factor of $0.22 \pm 0.01$ gives good agreement between the Monte Carlo and the data and is consistent with the value of 0.20 expected from cross section and efficiency calculations.

To check the normalization, we repeat the comparison between the estimated background and the data for two additional thresholds on the $\mathbb{E}_{T}: \boldsymbol{E}_{T}>25 \mathrm{GeV}$ and $\boldsymbol{E}_{T}$ $>35 \mathrm{GeV}$. The agreement is again very good, showing that the fractional backgrounds are well-understood (the multijet background varies by a factor of 6 , from 115 to 20 events, between the two thresholds). The number of $W+2 j$ events in the base data sample is estimated to be $11.7 \pm 1.8$ events.

\section{Total background}

Figures 14(a) and 14(b) show the $M_{T}^{e v}$ and $S_{T}^{12}$ distributions for the data sample and the background before the cut on $M_{T}^{e \nu}$. It is clear that we model the transverse mass distribution quite well up to $110 \mathrm{GeV} / c^{2}$. The $S_{T}^{12}$ distribution is also well-described by the MC except for the small systematic offset of the prediction relative to the data. The total background estimate after basic requirements is $17.8 \pm 2.1$ events, in agreement with the 14 events observed in the data.

\section{Efficiencies}

\section{Trigger efficiency}

Since events in the base data sample are required to have high electron $E_{T}$ and $\boldsymbol{E}_{T}$, the trigger requirements listed in Table VI are very efficient. The EM part of the trigger has an efficiency of $(99.5 \pm 0.5) \%$. 

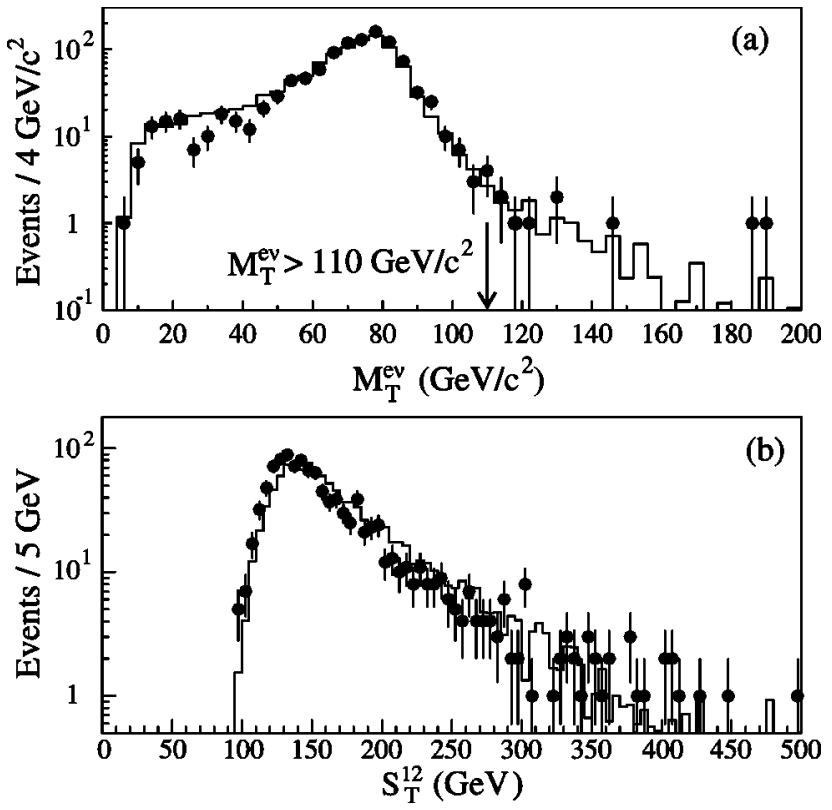

FIG. 14. Comparison of the (a) $M_{T}^{e v}$ and (b) $S_{T}^{12}$ distributions for the $e \nu j j$ data (points with error bars) and the predicted background (solid histogram) before imposing the $M_{T}^{e \nu}$ requirement.

\section{Efficiency of the minimal main ring veto}

As discussed in Sec. II, additional Main Ring (MR) trigger requirements can be applied offline to events collected using triggers with liberal MR requirements. For the $e \nu j j$ analysis, we apply a "minimal" MR veto to remove events that occurred during proton injection and when the proton bunch passed through the detector, while keeping events collected during the calorimeter recovery period. The efficiency of this veto is estimated using $Z+2 j$ data collected using triggers with looser $\mathrm{MR}$ requirements than in the triggers used in the $e \nu j j$ analysis. First, the MR requirements for the $e \nu j j$ triggers are applied to the $Z+2 j$ data. The efficiency of the minimal MR veto is then calculated by comparing the number of events in the $Z$-boson mass peak before and after the additional minimal MR veto requirements are applied. The efficiency of this veto is $(94 \pm 1) \%$ (i.e. $6 \%$ of the good events are removed along with a much larger percentage of background events). If the "calorimeter recovery" events were also removed, the efficiency would be reduced to about $90 \%$.

\section{Muon-veto efficiency}

The efficiency of the muon veto is estimated using a sample of $Z(\rightarrow e e)+2 j$ events. Except for the additional electron, these events have a topology similar to that of leptoquark events in the $e \nu j j$ channel and should have a similar random muon track rate. The calculation is done using the number of events in the $Z$-boson mass peak before and after application of the muon veto. Background under the $Z$ boson is subtracted using the standard side-band technique. The muon-veto efficiency is $(97 \pm 1) \%$.

\section{Electron identification efficiencies}

Using the efficiencies described in Sec. V D for the $e e j j$ channel, the overall electron identification efficiency for lep-

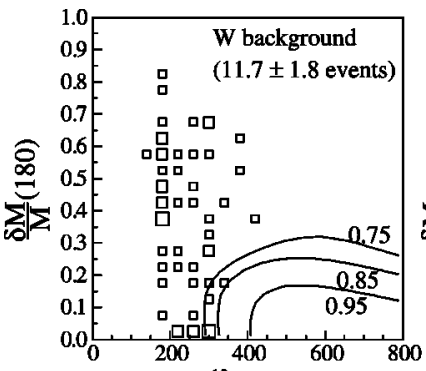

$\mathrm{S}_{\mathrm{T}}^{12}(\mathrm{GeV})$

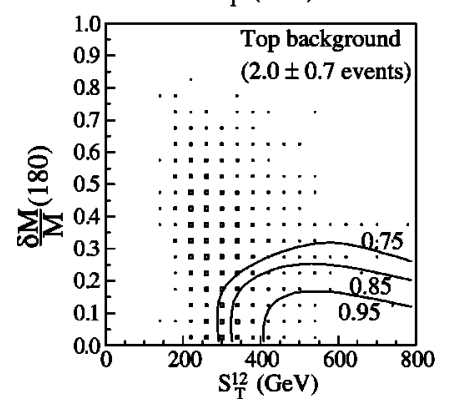

FIG. 15. Distributions of $(\delta M / M)(180)$ vs $S_{T}^{12}$ for the three individual backgrounds: (a) $W+2 j$ events, (b) multijet events, and (c) $t \bar{t}$ events. The curves show neural net contours for $\mathcal{D}_{\mathrm{NN}}(180)$ $=0.75,0.85$, and 0.95 .

toquark events in the $e \nu j j$ channel is $(61 \pm 4) \%$ in the CC and $(54 \pm 4) \%$ in the EC. Since $(93 \pm 1) \%$ of the electrons in the $e \nu j j$ final state are in the $\mathrm{CC}$, the total electron identification efficiency, including tracking and quality requirements, is $(60 \pm 3) \%$.

\section{E. Event selection optimization}

\section{Random grid search}

We use a random grid search based on the $M_{\mathrm{LQ}}=180$ $\mathrm{GeV} / c^{2} \mathrm{MC}$ sample to select the optimal variables and thresholds for the $e \nu j j$ channel. Many different variables and combinations of variables (see Sec. IV A) were tested for their efficiency in retaining the signal and rejecting the background. The inputs to the RGSEARCH program are the MC signal samples and the $W+2 j, t \bar{t}$, and multijet background samples described in Sec. VI C. The combinations of variables that have the most discriminating power are then used in the neural network analysis. The most powerful variables for separating leptoquark signals from the background are $S_{T}^{12}$ and $(\delta M / M)\left(M_{\mathrm{LQ}}\right)$ (see Sec. IV A).

\section{Neural network analysis}

We use a neural network with two input nodes [corresponding to the variables $S_{T}^{12}$ and $\left.(\delta M / M)\left(M_{\mathrm{LQ}}\right)\right]$, five hidden nodes, and one output node. A separate network is trained for each MC signal sample (with a desired network output $\left.\mathcal{D}_{\mathrm{NN}}\left(M_{\mathrm{LQ}}\right)=1\right)$ and the expected admixture of $W$ $+2 j, t \bar{t}$, and multijet background events (with desired $\left.\mathcal{D}_{\mathrm{NN}}\left(M_{\mathrm{LQ}}\right)=0\right)$. The expected rejection can be seen in Figs. 15 and 16. Figure 15 shows the two-dimensional distributions of $(\delta M / M)(180)$ versus $S_{T}^{12}$ for the three individual 

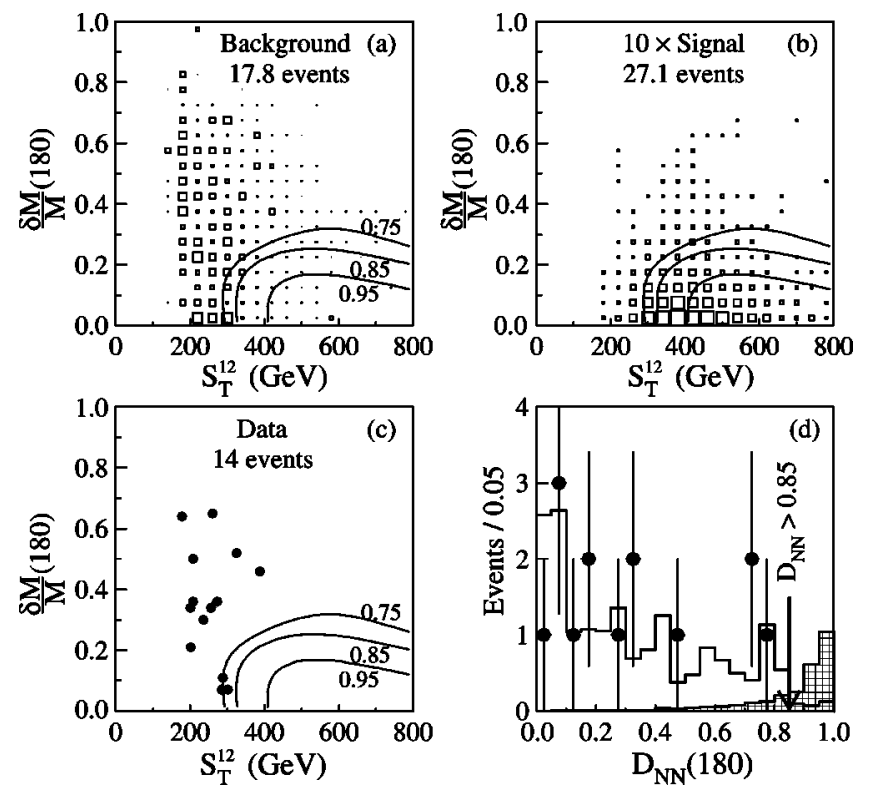

FIG. 16. Distributions of $(\delta M / M)(180)$ vs $S_{T}^{12}$ for (a) the total background, (b) ten times the expected signal from $180 \mathrm{GeV} / \mathrm{c}^{2}$ leptoquarks, and (c) the data. (d) The neural network discriminant for the signal (hatched histogram), the background (open histogram), and the data (points with error bars). The curves show neural net contours for $\mathcal{D}_{\mathrm{NN}}=0.95,0.85$, and 0.75 .

backgrounds. Figures 16(a)-16(c) show the same twodimensional distributions for the total background, simulated leptoquark events with $M_{\mathrm{LQ}}=180 \mathrm{GeV} / \mathrm{c}^{2}$, and the data. The contours corresponding to constant values of $\mathcal{D}_{\mathrm{NN}}(180)$ $=0.75,0.85$, and 0.95 demonstrate the level of separation achieved between the expected signal and the background. The distribution of $\mathcal{D}_{\mathrm{NN}}(180)$ for the data is compared with the predicted distributions for background and signal in Fig. 16(d). The data can be described by background alone. The highest value of $\mathcal{D}_{\mathrm{NN}}(180)$ observed in the base data sample is 0.79 .

Using the strategy described in Sec. IV B, we optimize the signal for a fixed background of approximately 0.4 events. In the low-mass range $\left(M_{\mathrm{LQ}} \leqslant 120 \mathrm{GeV} / c^{2}\right)$, where leptoquark production rates are high, requiring $S_{T}^{12}$ $>400 \mathrm{GeV}$ is sufficient and leads to a background of 0.60 \pm 0.27 events, consistent with the desired background level. For $M_{\mathrm{LQ}}>120 \mathrm{GeV} / c^{2}$, we use neural networks since they provide higher efficiency than an $S_{T}^{12}$ cut alone. For 180 $\mathrm{GeV} / c^{2}$ leptoquarks, approximately 0.4 background events are expected for $\mathcal{D}_{\mathrm{NN}}(180)>0.85$. We choose the $\mathcal{D}_{\mathrm{NN}}\left(M_{\mathrm{LQ}}\right)$ threshold to be a multiple of 0.05 rather than a value that yields exactly 0.4 background events; $\mathcal{D}_{\mathrm{NN}}(180)>0.85$ corresponds to a background of $0.29 \pm 0.25$ events. No events in the base data sample satisfy this criterion. Naturally, for leptoquark masses other than $180 \mathrm{GeV} / c^{2}$, the requirement on $\mathcal{D}_{\mathrm{NN}}\left(M_{\mathrm{LQ}}\right)$ is different. The expected background varies between 0.29 and 0.61 events and is listed in Table VII. No events from the base data sample pass any of these $\mathcal{D}_{\mathrm{NN}}\left(M_{\mathrm{LQ}}\right)$ thresholds.

Rectangular cuts of $S_{T}^{12}>350 \mathrm{GeV}$ and $(\delta M / M)(180)$ $<0.25$ yield a total background of 0.4 events. This also
TABLE VII. Efficiency, background, 95\% C.L. upper limit on the leptoquark production cross section, and NLO cross section multiplied by the branching fraction with $\mu=2 M_{\mathrm{LQ}}$ [22] for $\beta$ $=\frac{1}{2}$ as a function of leptoquark mass for the $e \nu j j$ channel.

\begin{tabular}{ccccc}
\hline \hline $\begin{array}{c}\text { Mass } \\
\left(\mathrm{GeV} / c^{2}\right)\end{array}$ & $\begin{array}{c}\text { Efficiency } \\
(\%)\end{array}$ & $\begin{array}{c}\text { Background } \\
(\text { Events })\end{array}$ & $\begin{array}{c}\sigma_{\text {limit }} \\
(\mathrm{pb})\end{array}$ & $\begin{array}{c}2 \beta(1-\beta) \sigma_{\mathrm{NLO}} \\
(\mathrm{pb})\end{array}$ \\
\hline 80 & $0.32 \pm 0.08$ & $0.60 \pm 0.27$ & 10.9 & 18.0 \\
100 & $1.15 \pm 0.21$ & $0.60 \pm 0.27$ & 2.6 & 5.34 \\
120 & $2.45 \pm 0.33$ & $0.60 \pm 0.27$ & 1.0 & 1.90 \\
140 & $6.65 \pm 0.96$ & $0.54 \pm 0.25$ & 0.43 & 0.77 \\
160 & $10.9 \pm 1.2$ & $0.61 \pm 0.27$ & 0.24 & 0.34 \\
180 & $14.7 \pm 1.2$ & $0.29 \pm 0.25$ & 0.18 & 0.16 \\
200 & $19.4 \pm 1.7$ & $0.43 \pm 0.27$ & 0.14 & 0.08 \\
220 & $21.5 \pm 1.7$ & $0.41 \pm 0.27$ & 0.12 & 0.04 \\
\hline \hline
\end{tabular}

leaves no events in the data sample, but the signal efficiency is approximately $10 \%$ lower for $M_{\mathrm{LQ}}=180 \mathrm{GeV} / c^{2}$.

\section{F. Check}

As a check of our understanding of the background, Fig. 17 shows the distribution of $\mathcal{D}_{\mathrm{NN}}(180)$ for the data and for the predicted background before the $M_{T}^{e v}$ cut. The agreement is acceptable.

\section{G. Signal studies}

\section{Systematic uncertainties}

The systematic uncertainty in the signal efficiency varies from $25 \%$ to $8 \%$ for $M_{\mathrm{LQ}}$ between 80 and $220 \mathrm{GeV} / \mathrm{c}^{2}$. The sources and sizes of the systematic uncertainties are given in Table VIII. The uncertainties due to the jet energy scale and initial and final state radiation are significantly lower than in the $e e j j$ analysis due to the use of $S_{T}^{12}$ rather than the alljets-based $S_{T}$ as a discriminator.

\section{Signal efficiency}

The signal detection efficiencies are calculated using simulated leptoquark events that pass the selection requirements; they are shown in Table VII. The errors in the signal efficiencies include uncertainties in trigger and particle-

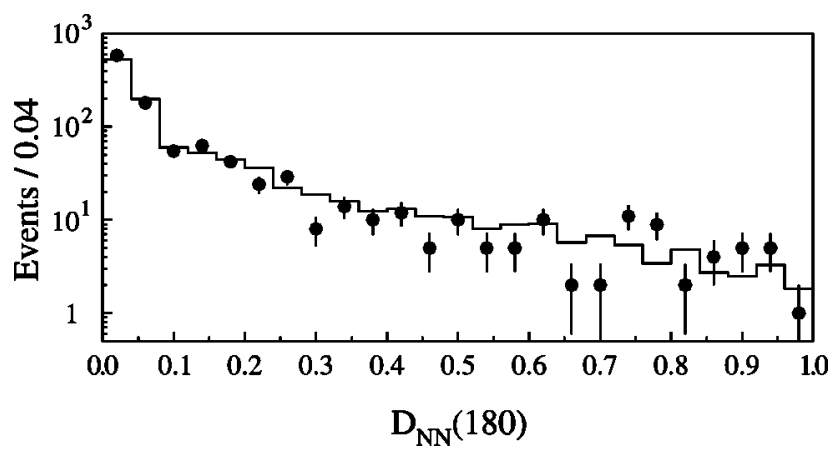

FIG. 17. Comparison of the $\mathcal{D}_{\mathrm{NN}}(180)$ distribution for the $e \nu j j$ data (points with error bars) and the predicted background (solid histogram) before the cut on $M_{T}^{e \nu}$. 
TABLE VIII. Systematic uncertainties in the signal for the $e \nu j j$ analysis.

\begin{tabular}{lc}
\hline \hline Source of systematics & Uncertainty $(\%)$ \\
\hline Particle identification & 5 \\
Smearing in the detector & 3 \\
Jet energy scale & $10-2\left(M_{\mathrm{LQ}}=80-220 \mathrm{GeV} / c^{2}\right)$ \\
Gluon radiation & 4 \\
PDF and $Q^{2}$ scale & 5 \\
Monte Carlo statistics & $25-3\left(M_{\mathrm{LQ}}=80-220 \mathrm{GeV} / c^{2}\right)$ \\
Luminosity & 5 \\
\hline Total & $25-8\left(M_{\mathrm{LQ}}=80-220 \mathrm{GeV} / c^{2}\right)$ \\
\hline \hline
\end{tabular}

identification efficiencies, the jet energy scale, effects of gluon radiation and parton fragmentation in the signal modeling, and finite MC statistics.

\section{H. Results from the $e \nu j j$ channel for scalar leptoquarks}

We obtain a $95 \%$ C.L. upper limit on the scalar leptoquark pair-production cross section for $\beta=\frac{1}{2}$ as a function of leptoquark mass. The results, based on a Bayesian analysis [43], are shown in Table VII. The statistical and systematic uncertainties in the efficiency, the integrated luminosity, and the background estimation are included in the limit calculation, all with Gaussian priors. The $95 \%$ C.L. upper limits on the cross section for scalar leptoquark pair production in the $e \nu j j$ channel, corrected for the branching fraction of $\beta=\frac{1}{2}$, for various leptoquark masses are plotted in Fig. 18 along with the NLO calculations [22]. The intersection of our limit with the lower edge of the theory band (renormalization scale $\mu=2 M_{\mathrm{LQ}}$ ) is at $0.38 \mathrm{pb}$, leading to a $95 \%$ C.L. lower limit on the leptoquark mass of $175 \mathrm{GeV} / c^{2}$.

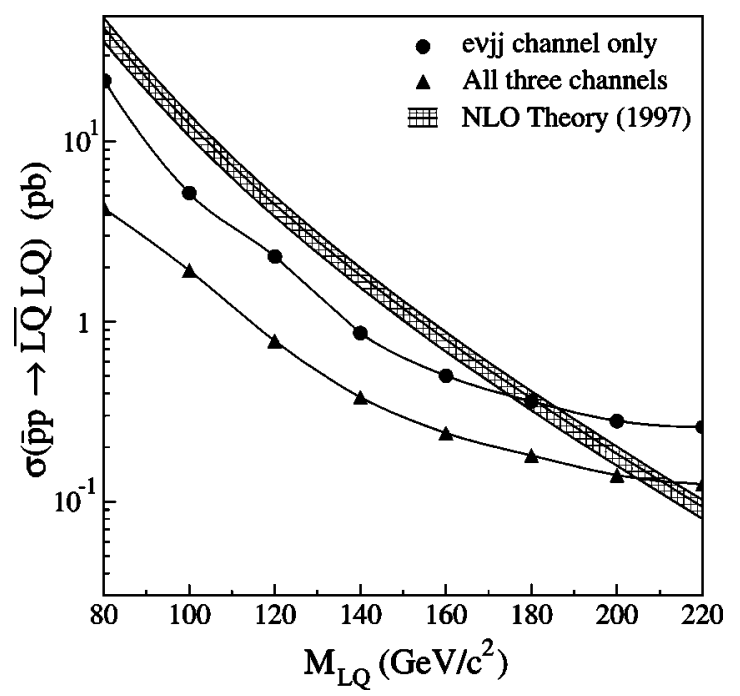

FIG. 18. The 95\% C.L. upper limits on the cross sections for scalar leptoquark pair production from the $e \nu j j$ channel, and for all three channels combined, for $\beta=\frac{1}{2}$, compared to the NLO prediction, as a function of leptoquark mass.

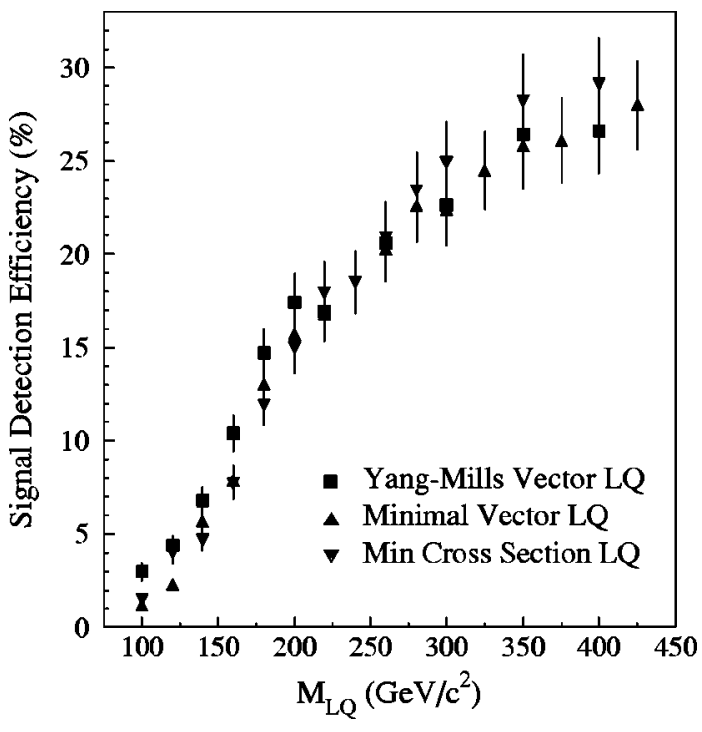

FIG. 19. The detection efficiency for vector leptoquarks in the $e \nu j j$ channel.

\section{Vector leptoquarks}

As in the case of the $e e j j$ channel, vector leptoquark events were generated for $M_{\mathrm{LQ}}$ between 100 and $425 \mathrm{GeV} /$ $c^{2}$ using a version of PYTHIA modified to include vector leptoquarks with different couplings. The distributions of the kinematic variables for scalar and vector leptoquarks are similar, and consequently, the same event selection is used for both analyses for $M_{\mathrm{LQ}} \leqslant 220 \mathrm{GeV} / \mathrm{c}^{2}$.

Neural networks for the $e \nu j j$ channel were trained on scalar leptoquark $\mathrm{MC}$ samples up to $M_{\mathrm{LQ}}=220 \mathrm{GeV} / \mathrm{c}^{2}$. Since vector leptoquark production cross sections are higher than scalar leptoquark cross sections, higher masses are of more interest. For vector leptoquarks with $M_{\mathrm{LQ}}>220 \mathrm{GeV} /$ $c^{2}$, we require $S_{T}^{12}>400 \mathrm{GeV}$. This variable is one of the inputs to the neural network and provides good signal identification efficiency and a background of $0.60 \pm 0.27$ events.

Again, the identification efficiencies for vector leptoquarks for the three couplings agree within their uncertainties, as shown in Fig. 19. Therefore, to reduce the statistical uncertainty in our analysis, we use the average identification efficiency of the three sets of MC events to set a single experimental limit on the cross section. As before, this limit is compared with the appropriate theoretical cross section for each coupling.

Figure 20 shows the experimental limits along with the three theoretical LO vector leptoquark cross sections [23] for the $e \nu j j$ channel for $\beta=\frac{1}{2}$ and $Q^{2}=M_{\mathrm{LQ}}^{2}$. For Yang-Mills coupling, the experimental lower limit on the vector leptoquark mass is $315 \mathrm{GeV} / c^{2}$, for $\beta=\frac{1}{2}$. For minimal vector coupling, the mass limit is $260 \mathrm{GeV} / c^{2}$ for $\beta=\frac{1}{2}$. For the coupling corresponding to the minimum cross section, the lower limit is $215 \mathrm{GeV} / c^{2}$ for $\beta=\frac{1}{2}$.

\section{VII. vvjj CHANNEL}

To analyze the $\nu \nu j j$ channel, we make use of our published search [47] for the supersymmetric partner of the top 


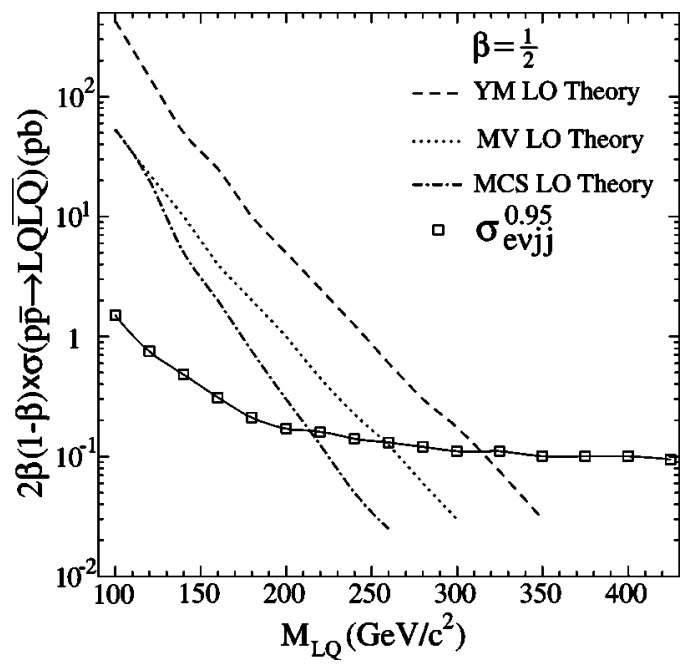

FIG. 20. The 95\% C.L. upper limits on the cross sections for vector leptoquark pair production from the $e \nu j j$ channel for $\beta=\frac{1}{2}$, and the LO predictions for the three couplings, as a function of leptoquark mass.

quark using just the 1992-1993 data sample. In that analysis, we searched for the pair production of top squarks that decay exclusively via a $c$ quark and the lightest neutralino, $\tilde{t}_{1}$ $\rightarrow c \tilde{\chi}_{1}^{0}$, resulting in a final state with $\mathbb{E}_{T}$ and two acolinear jets.

Approximately $75 \%$ of the data was collected using a trigger whose primary requirement was $\boldsymbol{E}_{T}>35 \mathrm{GeV}$ at level 2; the balance had a $\boldsymbol{E}_{T}$ threshold of $40 \mathrm{GeV}$. To ensure an unambiguous $\boldsymbol{E}_{T}$ measurement, events were required to have only one primary vertex, reducing the sample to single interactions with an integrated luminosity equivalent to approximately $7.4 \mathrm{pb}^{-1}$.

Events were required to have $\mathbb{E}_{T}>40 \mathrm{GeV}$, two jets with $E_{T}>30 \mathrm{GeV}$, and no isolated electrons or muons with $E_{T}$ $>10 \mathrm{GeV}$. In addition, the two leading jets were required to be acolinear $\left(90^{\circ}<\Delta \phi\left(j_{1}, j_{2}\right)<165^{\circ}\right)$, and the $\boldsymbol{E}_{T}$ was required not to be aligned with either the leading jet $\left(10^{\circ}\right.$ $\left.<\Delta \phi\left(j_{1}, \boldsymbol{E}_{T}\right)<125^{\circ}\right)$ or the third or fourth leading jets $\left[10^{\circ}<\Delta \phi\left(j_{3,4}, \mathbb{E}_{T}\right)\right]$. Three events survived the selection criteria, consistent with the estimated background of 3.5 \pm 1.2 events, primarily from $W / Z+$ jets production.

The efficiencies of the event selection for scalar leptoquarks with $M_{\mathrm{LQ}}$ from 50 to $200 \mathrm{GeV} / \mathrm{c}^{2}$ are calculated using signal $\mathrm{MC}$ events generated with the ISAJET generator and processed through the GEANT-based detector simulation. The systematic errors in the signal acceptance are calculated as in Ref. [47]. The efficiencies, background, and cross section limits are shown in Table IX. This analysis yields the limit $M_{\mathrm{LQ}}>79 \mathrm{GeV} / c^{2}$ at the $95 \%$ C.L. for $\beta=0$.

The identification efficiency for vector leptoquark (generated using PYTHIA) and scalar leptoquark events with $M_{\mathrm{LQ}}$ $=200 \mathrm{GeV} / c^{2}$ are identical, within errors. Based on this comparison, and similar comparisons in the eejj and $e \nu j j$ channels, we use the experimental limit for scalar leptoquarks for vector leptoquarks in the $\nu \nu j j$ channel. Comparison with the theoretical cross sections leads to $95 \%$ C.L.
TABLE IX. Efficiency, background, 95\% C.L. upper limit on the leptoquark pair production cross section, and the NLO cross section with $\mu=2 M_{\mathrm{LQ}}$ [22] for $\beta=0$ as a function of leptoquark mass for the $\nu \nu j j$ channel.

\begin{tabular}{clccc}
\hline \hline $\begin{array}{c}\text { Mass } \\
\left(\mathrm{GeV} / c^{2}\right)\end{array}$ & $\begin{array}{c}\text { Efficiency } \\
(\%)\end{array}$ & $\begin{array}{c}\text { Background } \\
(\text { events })\end{array}$ & $\begin{array}{c}\sigma_{\text {limit }} \\
(\mathrm{pb})\end{array}$ & $\begin{array}{c}\sigma_{\mathrm{NLO}} \\
(\mathrm{pb})\end{array}$ \\
\hline 50 & $0.446_{-0.107}^{+0.096}$ & $3.49 \pm 1.17$ & 328 & 406 \\
60 & $1.11 \pm 0.16$ & $3.49 \pm 1.17$ & 77.0 & 162 \\
80 & $2.15_{-0.22}^{+0.23}$ & $3.49 \pm 1.17$ & 37.7 & 36.0 \\
100 & $3.90 \pm 0.30$ & $3.49 \pm 1.17$ & 21.0 & 10.7 \\
120 & $4.62_{-0.32}^{+0.30}$ & $3.49 \pm 1.17$ & 17.6 & 3.81 \\
140 & $6.07 \pm 0.34$ & $3.49 \pm 1.17$ & 13.2 & 1.54 \\
160 & $6.15 \pm 0.34$ & $3.49 \pm 1.17$ & 13.0 & 0.68 \\
200 & $6.36_{-0.36}^{+0.35}$ & $3.49 \pm 1.17$ & 12.6 & 0.16 \\
\hline \hline
\end{tabular}

limits of $M_{\mathrm{LQ}}>206,154$, and $144 \mathrm{GeV} / c^{2}$ for Yang-Mills, minimal vector, and minimum cross section couplings, respectively, for $\beta=0$.

\section{GAP IN THE LIMIT FOR SCALAR LEPTOQUARKS}

In our analysis of the $e e j j$ and $e \nu j j$ channels, we use MC samples of leptoquarks with $M_{\mathrm{LQ}} \geqslant 80 \mathrm{GeV} / c^{2}$, but our analysis is optimized for leptoquarks with masses near 200 $\mathrm{GeV} / c^{2}$. From the $e \nu j j$ analysis, we exclude $\beta>0.13$ for $M_{\mathrm{LQ}}=80 \mathrm{GeV} / c^{2}$. The mass limit from the $\nu \nu j j$ channel for $\beta=0.13$ is approximately $75 \mathrm{GeV} / \mathrm{c}^{2}$, leaving a small gap in our limit.

To fill this gap, we examine further the 14 events in the base data sample in the $e \nu j j$ analysis. Making the very conservative assumption that all 14 events are due to leptoquark pair production, the 95\% C.L. upper limit on the cross section multiplied by the branching fraction and efficiency is $0.20 \mathrm{pb}$. This permits us to extend our exclusion region to include $0.09 \leqslant \beta \leqslant 0.91$ for $M_{\mathrm{LQ}}=80 \mathrm{GeV} / c^{2}$ and $0.05 \leqslant \beta$ $\leqslant 0.95$ for $M_{\mathrm{LQ}}=75 \mathrm{GeV} / c^{2}$. To obtain the efficiency for $M_{\mathrm{LQ}}=75 \mathrm{GeV} / \mathrm{c}^{2}$, we scale the efficiency found for higher $M_{\mathrm{LQ}}$.

\section{COMBINED RESULTS}

Combining [43] the limits from the $e e j j, e \nu j j$, and $\nu \nu j j$ channels, we obtain 95\% C.L. upper limits on the leptoquark pair-production cross section as a function of leptoquark mass and $\beta$. The cross-section limits for $\beta=\frac{1}{2}$ are shown in Fig. 18 for scalar leptoquarks and in Fig. 21 for vector leptoquarks. Table $\mathrm{X}$ lists the mass limits for $\beta=1, \frac{1}{2}$, and 0 for

TABLE X. Limits on the masses of first-generation leptoquarks.

Scalar Minimum cross section Minimal vector Yang-Mills

\begin{tabular}{ccccc}
$\beta$ & $\left(\mathrm{GeV} / c^{2}\right)$ & $\left(\mathrm{GeV} / c^{2}\right)$ & $\left(\mathrm{GeV} / c^{2}\right)$ & $\left(\mathrm{GeV} / c^{2}\right)$ \\
\hline 1 & 225 & 246 & 292 & 345 \\
$\frac{1}{2}$ & 204 & 233 & 282 & 337 \\
0 & 79 & 144 & 159 & 206 \\
\hline
\end{tabular}




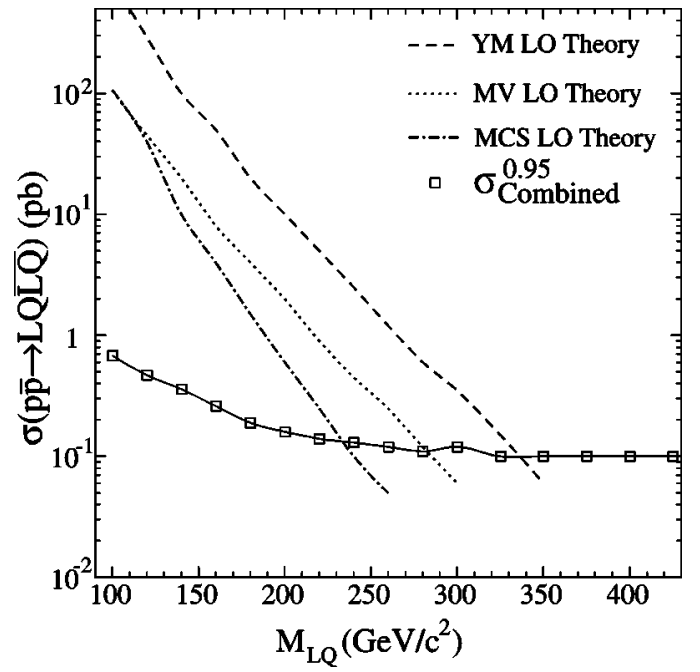

FIG. 21. The 95\% C.L. upper limits on cross sections for vector leptoquark pair production from all three channels combined for $\beta=\frac{1}{2}$, and the LO predictions, as a function of leptoquark mass.

the types of leptoquarks studied. The lower limits on the mass of scalar leptoquarks as a function of $\beta$, for all three channels combined, as well as for the individual channels, are shown in Fig. 22. Figure 23 shows the exclusion contours from the individual channels and the combined result for vector leptoquarks with Yang-Mills coupling. Figure 24 shows the overall exclusion contours for the three vector couplings.

\section{CONCLUSIONS}

We have presented $95 \%$ C.L. upper limits on the pair production of leptoquarks that decay to the eejj, e $e j j$, and $\nu \nu j j$ final states. For scalar leptoquarks, the limits on the cross section provide lower limits on the scalar leptoquark

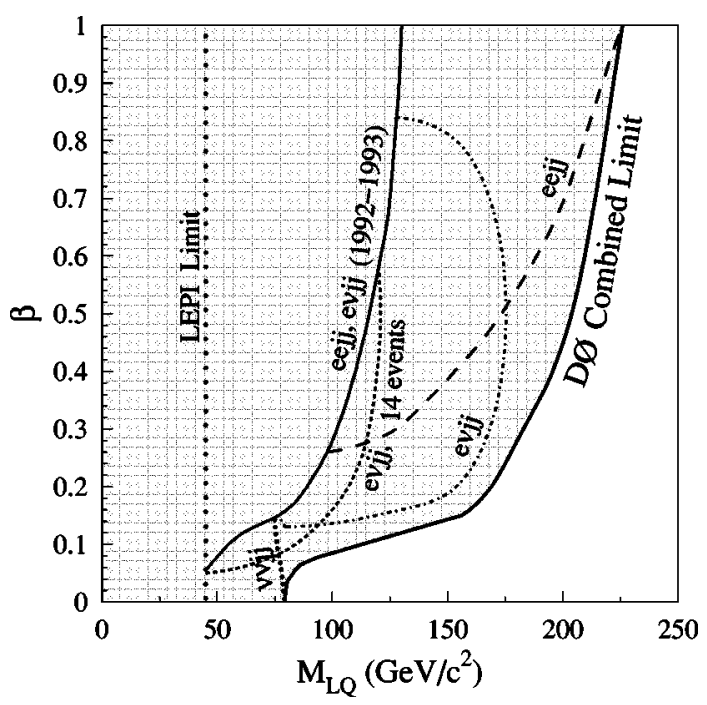

FIG. 22. The 95\% C.L. lower limit on the mass of firstgeneration scalar leptoquarks as a function of $\beta$ for the individual $e e j j$, $e \nu j j$, and $\nu \nu j j$ channels, and for the combined analysis.

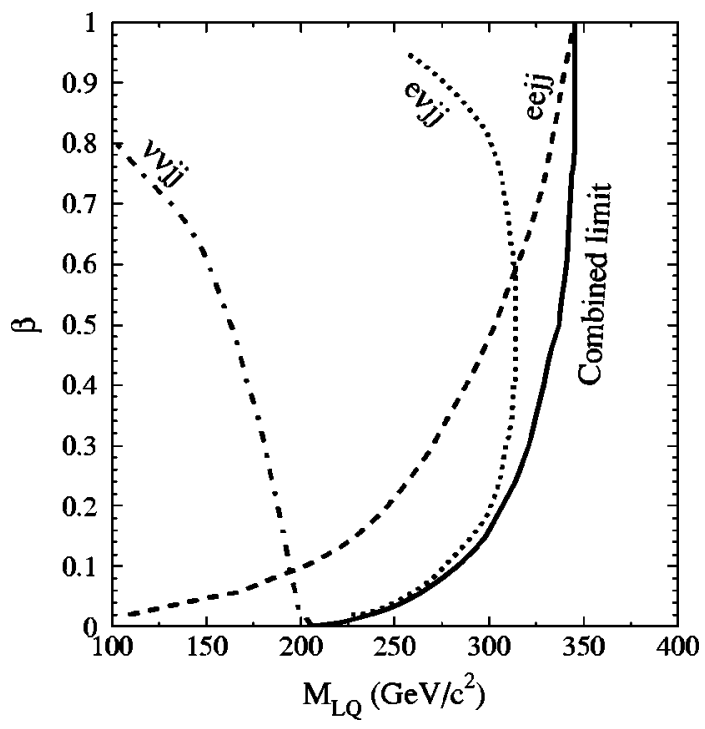

FIG. 23. The 95\% C.L. lower limit, as a function of $\beta$, on the mass of first-generation vector leptoquarks with Yang-Mills couplings from the individual $e e j j, e \nu j j$, and $\nu \nu j j$ channels and for the combined analysis.

mass of $225 \mathrm{GeV} / c^{2}$ for $\beta=1,204 \mathrm{GeV} / c^{2}$ for $\beta=\frac{1}{2}$, and 79 $\mathrm{GeV} / c^{2}$ for $\beta=0$. We have also set mass limits for vector leptoquarks for different couplings and have presented exclusion contours on $\beta$ and $M_{\mathrm{LQ}}$. At the 95\% C.L., our results exclude an interpretation of the HERA high- $Q^{2}$ excess as $s$-channel scalar leptoquark production for $M_{\mathrm{LQ}}<200 \mathrm{GeV} /$ $c^{2}$ and $\beta>0.4$. These results can be also used to set limits on the pair production of any heavy scalar particle that decays into a lepton and a quark as expected in a variety of models and to restrict any new leptoquark models containing additional fermions [48].

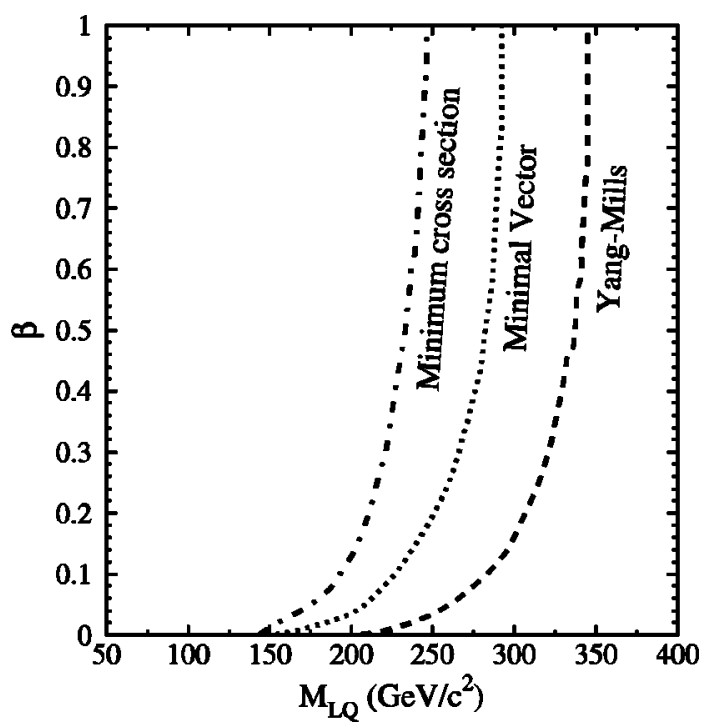

FIG. 24. The $95 \%$ C.L. lower limits on $M_{\mathrm{LQ}}$ as a function of $\beta$ for first-generation vector leptoquarks with Yang-Mills, minimal vector, and minimum cross section couplings from the $e e j j, e \nu j j$, and $\nu \nu j j$ channels combined. 


\section{ACKNOWLEDGMENTS}

We are grateful to M. Krämer for helpful discussions and for providing us with detailed cross section information. We also thank J. L. Hewett and T. G. Rizzo for many valuable discussions, and C. Grosso-Pilcher for sharing results from CDF with us and working on the combination of our limits. Finally, we thank the staffs at Fermilab and collaborating institutions, and acknowledge support from the U.S. Department of Energy and National Science Foundation, Commis- sariat à L'Energie Atomique and CNRS/Institut National de Physique Nucléaire et de Physique des Particules (France), Ministry for Science and Technology and Ministry for Atomic Energy (Russia), CAPES and CNPq (Brazil), Departments of Atomic Energy and Science and Education (India), Colciencias (Colombia), CONACyT (Mexico), Ministry of Education and KOSEF (Korea), CONICET and UBA-CyT (Argentina), The Foundation for Fundamental Research on Matter (The Netherlands), PPARC (United Kingdom), Ministry of Education (Czech Republic), and the A. P. Sloan Foundation.
[1] D. Acosta and S. Blessing, Annu. Rev. Nucl. Part. Sci. 49, 389 (1999), and references therein.

[2] H1 Collaboration, I. Abt et al., Nucl. Phys. B396, 3 (1993).

[3] H1 Collaboration, T. Ahmed et al., Z. Phys. C 64, 545 (1994).

[4] H1 Collaboration, S. Aid et al., Phys. Lett. B 353, 578 (1995).

[5] H1 Collaboration, S. Aid et al., Phys. Lett. B 369, 173 (1996).

[6] H1 Collaboration, C. Adloff et al., Eur. Phys. J. C 11, 447 (1999).

[7] H1 Collaboration, C. Adloff et al., Phys. Lett. B 479, 358 (2000).

[8] ZEUS Collaboration, M. Derrick et al., Phys. Lett. B 306, 173 (1993).

[9] ZEUS Collaboration, M. Derrick et al., Z. Phys. C 73, 613 (1997).

[10] ZEUS Collaboration, J. Breitweg et al., Phys. Rev. D 63, 052002 (2001).

[11] OPAL Collaboration, G. Abbiendi et al., Eur. Phys. J. C 13, 15 (2000).

[12] DELPHI Collaboration, P. Abreu et al., Phys. Lett. B 446, 62 (1999).

[13] L3 Collaboration, B. Adeva et al., Phys. Lett. B 261, 169 (1991).

[14] ALEPH Collaboration, D. Decamp et al., Phys. Rep. 216, 253 (1992).

[15] DФ Collaboration, B. Abbott et al., Phys. Rev. Lett. 79, 4321 (1997).

[16] DØ Collaboration, B. Abbott et al., Phys. Rev. Lett. 80, 2051 (1998).

[17] CDF Collaboration, F. Abe et al., Phys. Rev. Lett. 79, 4327 (1997)

[18] H1 Collaboration, C. Adloff et al., Z. Phys. C 74, 191 (1997).

[19] ZEUS Collaboration, J. Breitweg et al., Z. Phys. C 74, 207 (1997).

[20] J. L. Hewett and T. Rizzo, Phys. Rev. D 56, 5709 (1997), and references therein.

[21] ZEUS Collaboration, J. Breitweg et al., Eur. Phys. J. C 11, 427 (1999).

[22] M. Krämer, T. Plehn, M. Spira, and P. M. Zerwas, Phys. Rev. Lett. 79, 341 (1997).

[23] J. Blümlein, E. Boos, and A. Kryukov, Z. Phys. C 76, 137 (1997).

[24] DØ Collaboration, S. Abachi et al., Nucl. Instrum. Methods Phys. Res. A 338, 185 (1994).

[25] Pseudorapidity is defined as $\eta=-\ln \tan (\theta / 2)$, where $\theta$ is the polar angle measured with respect to the proton beam and relative to the $z$ position of the interaction vertex. We also use a variable called "detector pseudorapidity," $\eta_{\text {det }}$, for which $\theta$ is measured relative to the center of the detector, $z=0$.

[26] DØ Collaboration, S. Abachi et al., Phys. Rev. D 52, 4877 (1995).

[27] DØ Collaboration, B. Abbott et al., Phys. Rev. D 58, 052001 (1998).

[28] DØ Collaboration, B. Abbott et al., Phys. Rev. D 64, 032003 (2001).

[29] DØ Collaboration, B. Abbott et al., Phys. Rev. Lett. 80, 2063 (1998).

[30] DØ Collaboration, S. Abachi et al., Phys. Rev. Lett. 77, 3309 (1996).

[31] DØ Collaboration, B. Abbott et al., Phys. Rev. Lett. 79, 1203 (1997).

[32] D $\emptyset$ Collaboration, S. Abachi et al., Phys. Rev. D 57, 589 (1998).

[33] DØ Collaboration, B. Abbott et al., Phys. Rev. Lett. 79, 1197 (1997).

[34] DØ Collaboration, B. Abbott et al., Phys. Rev. D 60, 012001 (1999).

[35] DØ Collaboration, B. Abbott et al., Phys. Rev. Lett. 83, 1908 (1999).

[36] V. Barger and R. Phillips, Collider Physics (Addison-Wesley, Reading, MA, 1987).

[37] N. Amos et al., in Proceedings of the International Conference on Computing in High Energy Physics (CHEP'95), edited by R. Shellard and T. Nguyen (World Scientific, Singapore, 1996), p. 215.

[38] L. Lönnblad et al., Comput. Phys. Commun. 81, 185 (1994), we used JETNET v3.0.

[39] D $\emptyset$ Collaboration, P. Bhat, in Proceedings of the 10th Topical Workshop on Proton-Antiproton Collider Physics, edited by R. Raja and J. Yoh (AIP, New York, 1995), p. 308, and references therein.

[40] F. Paige and S. Protopopescu, BNL Note 38304 (1986), we used ISAJET v7.22 with CTEQ2L parton distribution functions.

[41] G. Marchesini et al., Comput. Phys. Commun. 67, 465 (1992), and we used HERWIG v5.7.

[42] O. Dahl et al., "SQUAW Kinematic Fitting Program," LBL Group A P-126 (1968).

[43] I. Bertram et al., "A Recipe for the Construction of Confidence Limits," Fermilab TM-2104 (1998).

[44] Leptoquark Limit Combination Working Group, "Combined Limits on First Generation Leptoquarks from the CDF and D $\emptyset$ Experiments," hep-ex/9810015. 
[45] T. Sjöstrand, Comput. Phys. Commun. 82, 74 (1994), we used PYTHIA v5.7.

[46] F. Berends, H. Kuijf, B. Tausk, and W. Giele, Nucl. Phys. B357, 32 (1991).
[47] DФ Collaboration, S. Abachi et al., Phys. Rev. Lett. 76, 2222 (1996).

[48] J. L. Hewett and T. Rizzo, Phys. Rev. D 58, 055005 (1998). 